\title{
Performance Analysis of Gradient-Based Nash Seeking Algorithms Under Quantization
}

\author{
Ehsan Nekouei Member, IEEE, Girish N. Nair, Senior Member, IEEE, and Tansu Alpcan Senior Member, IEEE
}

\begin{abstract}
This paper investigates the impact of quantized inter-agent communications on the asymptotic and transient behavior of gradient-based Nash-seeking algorithms in noncooperative games. Using the information-theoretic notion of entropy power, we establish a universal lower bound on the asymptotic rate of exponential mean-square convergence to the Nash equilibrium (NE). This bound depends on the inter-agent data rate and the local behavior of the agents' utility functions, and is independent of the quantizer structure. Next, we study transient performance and derive an upper bound on the average time required to settle inside a specified ball around the NE, under uniform quantization. Furthermore, we establish an upper bound on the probability that agents' actions lie outside this ball, and show that this bound decays double-exponentially with time. Finally, we propose an adaptive quantization scheme that allows the gradient algorithm to converge to the NE despite quantized inter-agent communications.
\end{abstract}

Index Terms-

\section{INTRODUCTION}

\section{A. Motivation}

Game theory has proved to be of ubiquitous importance in engineering and can be used to analyze numerous problems, e.g., power control in wireless networks [1], wind energy harvesting, sensor coverage[2] etc. In non-cooperative games, multiple agents aim to maximize individual utility functions by taking actions that are not necessarily coordinated with one another. The Nash equilibrium $(N E)$ is one of the most important solution concepts in such games. It is a point in the action space at which no agent can increase its own utility by unilaterally changing its action.

The problem of finding Nash equilibria is an active research area that has attracted much attention, e.g., see [3] and [4] and references therein. Gradient-based equilibrium-seeking (ES) algorithms are popular techniques for finding the NE of games with continuous action spaces and differentiable utility functions. In such algorithms, each agent modifies its current action according to the partial derivative of its utility function with respect to its action. The computation of this derivative implicitly requires communication between agents, since it typically depends on the actions of other agents.

In this paper, we consider a non-cooperative game in which actions are communicated between agents over channels with finite data rates or capacities. As a result, the agents' actions cannot be transmitted exactly, but must instead be quantized

\footnotetext{
Department of Electrical and Electronic Engineering, The University of Melbourne, VIC 3010, Australia. E-mails: \{ehsan.nekouei, gnair, tansu.alpcan\}@unimelb.edu.au

This work was supported by the Australian Research Council's Discovery Projects funding scheme (DP140100819).
}

using a finite number of bits per time instant. It is known that such data rate limitations can have detrimental impacts on the performance of control and optimization algorithms. For example, a communication channel deployed in a feedback control system can destabilize the system if its data rate is too low, e.g., see [13], [14] and [17]. In cooperative distributed optimization, quantization affects convergence speeds negatively [8], [9].

In this paper, we investigate the effect of quantized communication on gradient-based, Nash-seeking algorithms. More specifically, we are interested in the following questions: $(i)$ How does the communication data rate generally affect the convergence speeds achievable by ES algorithms? ( $i i)$ Given a uniform quantization scheme, on average how many timesteps are required for the ES algorithm to settle inside a ball around the NE? ( iii) Given a uniform quantization scheme, what is the probability that agents' actions lie outside this ball at a given time?

\section{B. Related Work}

Cooperative multi-agent optimization problems have been widely studied e.g., see [5]-[7] and references therein. However, the effect of quantized inter-agent communication in such problems is a relatively new research area. Nedić et al. [8] proposed an averaging-based algorithm for solving a multi-agent convex optimization problem, in which agents seek to cooperatively minimize a global cost comprising a sum of individual objective functions. They studied the convergence rate of the proposed algorithm under infinitelevel, uniform quantization. Rabbat et al. [9] studied a convex optimization problem in which a set of agents cooperate to minimize a sum of individual objective functions, also under uniform quantization. In each iteration of their algorithm, only one agent updates the decision variables based on its local information, and then passes its estimate of decision variables to the next agent. The authors in [10] considered the problem of minimizing an upper bound on the distortion due to quantization in distributed iterative algorithms. They established the optimality of different quantization structures under various distortion measures.

It should be noted that, in game formulations, the objective functions of agents are usually non-convex, and agents seek to find the NE, which is a fundamentally different concept from the optimal solution of a convex optimization problem.

The convergence behavior of consensus algorithms under quantized communications has also been investigated in the literature. In a consensus problem, a group of agents seek to 
arrive at a common state value. Kashyap et al. considered an average-consensus problem in which actions of agents are limited to integer numbers. They proposed a gossipbased algorithm for achieving consensus and analyzed its convergence behavior. Dong et al. [11] analyzed the error performance of an average-consensus protocol, in a multiagent consensus problem, wherein each agent, first, quantizes its state value using a uniform quantizer, then, it transmits the quantized version of its state to other agents via a communication graph. The authors in [12] studied the convergence rate of an average-consensus algorithm under time-varying/timeinvariant communication graphs and quantized message passing between agents. Nedić et al. [16] investigated the effect of quantization and time-varying communication topologies on the convergence time of average-consensus algorithms. In the case where agents have local linear time-invariant dynamics, You and Xie [17] derived necessary and sufficient conditions on the communication graph and the data rate between agents for consensability.

Finally, we note that the impact of quantization on the stability of feedback control systems has been extensively studied in the literature. As this is not the main direction of our research, interested readers are referred to [13], [14] and references therein for a thorough investigation of this topic.

\section{Contributions}

This paper investigates the impact of quantized inter-agent communications on the asymptotic and transient performance of non-cooperative games. It is assumed that the game has a unique Nash equilibrium (NE) and that agents employ a gradient-based equilibrium-seeking (ES) algorithm to reach it, beginning from a random initial condition. As each agent's gradient typically depends on other agents' actions, the agents require some knowledge of one another's actions. In this paper, it is assumed that the inter-agent communication is performed through a complete communication graph, i.e., each agent broadcasts its action to all other agents. Although each agent knows its own actions exactly, it has access to only quantized versions of other agents' actions, due to communication limitations.

There are four contributions in this paper. Firstly, we establish a universal lower bound on the rate of exponential meansquare convergence to the NE (Theorem 1). Our results show that the convergence rate is limited by the behavior of utility functions of agents around the NE and also by the aggregate communication data rate between agents. Importantly, this result is independent of the choice of quantization scheme. Our proof uses the information-theoretic notion of differential entropy power, which has been previously used to study feedback control under communication constraints; see e.g. [23] and [24].

Next, we consider two performance measures to analyze the transient behavior of a gradient-based ES algorithm under a uniform quantizer: $(i)$ the expected number of time-steps required for all agents' actions to settle inside a ball of radius $r$ centered at the NE, and (ii) the probability that agents' actions, at any given time, lie outside this ball. We prove upper bounds (Theorems 2,3) on these criteria and show that, as the time-index becomes large, the probability of lying outside the specified ball decays double-exponentially to zero. Based on this analysis, we then propose an adaptive quantization scheme that achieves asymptotic convergence to the NE.

There are several distinctions between this paper and the related literature discussed in the previous subsection. Most obviously, our agents do not cooperate to minimize a global criterion or agree on a common value, but instead seek to maximize individual objective functions. This introduces significant differences in formulation and technique. In particular, the convergence analysis techniques of [8], [9] cannot be applied because they rely on the objective function never dropping below its optimal value, which fact is used to bound the distance from the minimum. In contrast, in the gametheoretic setting here each agent's objective function value at any time step can be either greater or smaller than its value at the NE. In addition, we study both transient performance and asymptotic convergence rates, and our analysis of mean-square convergence rates is not restricted to uniform quantization schemes.

Our asymptotic result provides guidelines on the required data-rate for achieving a particular speed of exponential convergence to the NE. Also, our transient results give rise to bounds on the required number of quantization levels for achieving an average settling time (inside a ball around the NE).

This paper is organized as follows. In the next section, we describe our system model and assumptions. In section III, we state our main results regarding the asymptotic and transient behaviors of the ES algorithm under quantized interagent communications. We present numerical results in section IV, and conclude the paper in section V. To aid the flow of the paper, we relegate all proofs to the Appendices.

\section{SySTEM MODEL}

We consider a game with $M$ agents, indexed by $i \in \mathcal{M}:=$ $\{1, \cdots, M\}$. Let $x^{i} \in \mathbb{R}$ be the action of the $i$-th agent, $\boldsymbol{x}^{-i}:=\left[x^{1}, \cdots, x^{i-1}, x^{i+1}, x^{M}\right]^{\top} \in \mathbb{R}^{M-1}$, the vector of all agents actions except the $i$-th, and $U_{i}\left(x^{i}, \boldsymbol{x}^{-i}\right) \in \mathbb{R}$ the utility of the $i$-th agent. We refer to this game as $\mathcal{G}=$ $\left\langle\mathcal{M},\left\{x^{i}\right\}_{i},\left\{U_{i}(\cdot)\right\}_{i}\right\rangle$. We assume that each utility function $U_{i}\left(x^{i}, \boldsymbol{x}^{-i}\right)$ is twice continuously differentiable and concave with respect to $x^{i}$.

Ideally, each agent in the game would like to make its own utility as large as possible. However, since the global maximizers of the utility functions will not generally coincide, a compromise is needed. This is provided by the Nash equilibrium $(N E)$, which is one of the most widely accepted solution concepts for non-cooperative games. If all agents play their NE strategies, denoted by $x_{\mathrm{NE}}^{i}, i \in \mathcal{M}$, then no agent can increase its individual utility by unilaterally changing its action, i.e.

$$
x_{\mathrm{NE}}^{i}=\arg \max _{x^{i}} \quad U_{i}\left(x^{i}, \boldsymbol{x}_{\mathrm{NE}}^{-i}\right), \forall i \in \mathcal{M} .
$$


This notion is relevant for non-cooperative games, in which agents do not form teams to take joint actions, but rather act as individuals.

Throughout this paper, we assume that the game admits a unique NE. This can be easily satisfied by imposing some additional mild conditions on the utility functions of agents, e.g., see [18] and [19]. For example, in [18], it was shown that if the matrix $J\left(\nabla \sum_{i} r_{i} U_{i}\left(x^{i}, \boldsymbol{x}^{-i}\right)\right)+J\left(\nabla \sum_{i} r_{i} U_{i}\left(x^{i}, \boldsymbol{x}^{-i}\right)\right)^{\top}$ is negative definite for some $r_{i}>0 \quad \forall i$, then the game admits a unique Nash equilibrium. Here, $J\left(\nabla \sum_{i} r_{i} U_{i}\left(x^{i}, \boldsymbol{x}^{-i}\right)\right)$ represents the Jacobian of $\nabla \sum_{i} r_{i} U_{i}\left(x^{i}, \boldsymbol{x}^{-i}\right)$ with respect to $\boldsymbol{x}$. Games arising in many engineering applications naturally admit a unique NE, e.g., see [20] and [21].

\section{A. Gradient-based Equilibrium Seeking with Quantized Com- munication}

Gradient-based, equilibrium seeking (ES) algorithms are among the most popular iterative techniques for finding the $\mathrm{NE}$ of a game with continuous action spaces and differentiable utility functions. In the absence of quantization, such algorithms take the general form

$x_{k+1}^{i}=x_{k}^{i}+\mu_{k} \frac{\partial}{\partial x^{i}} U_{i}\left(x_{k}^{i}, \boldsymbol{x}_{k}^{-i}\right), k \in \mathbb{N}_{0}:=\{0,1,2 \ldots\}$

where $x_{k}^{i}$ is the action of the $i$-th agent at time $k, \boldsymbol{x}_{k}^{-i}$ is the vector of all agent actions at time $k$ except the $i$-th, and $\mu_{k}>0$ is a time-varying step size.

In order to implement this update rule, each agent does not need to know other agents' utility functions, which may be kept private, but only their latest actions. However, agents in a distributed game are often located far from each other, e.g. power plants competing in a wholesale electricity market for maximizing their individual profits. The long distance between agents, combined with finite transmission power and bandwidth, limit the communication capacity between agents. Consequently, the agents in distributed games cannot transmit their actions with infinite resolution, but instead exchange quantized versions that are representable with finite numbers of bits.

Assuming that each $i$-th agent knows its own action $x_{k}^{i}$ perfectly, let $D_{i, k}\left(x_{k}^{i}\right) \in \mathcal{A}_{i, k}$ represent the quantized action broadcast by it to all other agents at time $k$. Here $\mathcal{A}_{i, k} \subset \mathbb{R}$ is a finite set and $\left|\mathcal{A}_{i, k}\right|$ is the number of quantization levels used by the $i$-th agent at time $k$. A large value of $\left|\mathcal{A}_{i, k}\right|$ implies that the $i$-th agent transmits its action with high precision, whereas a low value reflects poor communication capacity and low precision. Let $D_{k}\left(\boldsymbol{x}_{k}\right) \in \mathcal{A}_{k}$ represent the component-wise quantized version of the vector $\boldsymbol{x}_{k}$, that is $D_{k}\left(\boldsymbol{x}_{k}\right)=\left[D_{i, k}\left(x_{k}^{i}\right)\right]_{i}^{\top}$. Note that $\log \left|\mathcal{A}_{k}\right|$ denotes the aggregate number of bits used by agents to represent their actions in the $k$-th iteration, where $\left|\mathcal{A}_{k}\right|=\prod_{i=1}^{M}\left|\mathcal{A}_{i, k}\right|$. With a slight abuse of notation, let $\mathcal{D}_{k}\left(\boldsymbol{x}_{k}^{-i}\right)$ denote the quantized version of the vector $\boldsymbol{x}_{k}^{-i}$. The ES algorithm with quantization then takes the form

$$
x_{k+1}^{i}=x_{k}^{i}+\mu_{k} \frac{\partial}{\partial x^{i}} U_{i}\left(x_{k}^{i}, \mathcal{D}_{k}\left(\boldsymbol{x}_{k}^{-i}\right)\right), \quad \forall i \in \mathcal{M} .
$$

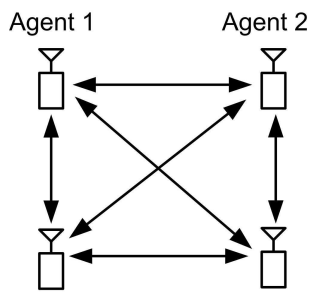

Agent 2

(a) Fully connected communication topology

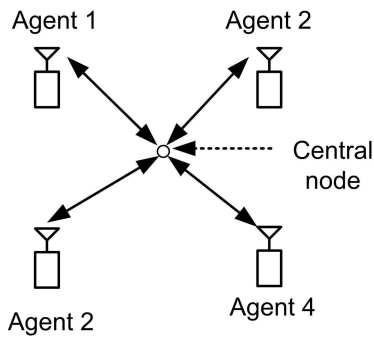

(b) Star communication topology
Fig. 1. A pictorial example of the fully connected and star communication topologies.

We refer to $\mathcal{D}=\left\{D_{k}\right\}_{k=0}^{\infty}$ as a quantization scheme. Given $\mathcal{D}$, the average aggregate data rate per unit time is defined as

$$
R_{\mathcal{D}}:=\limsup _{k \longrightarrow \infty} \frac{1}{k} \sum_{j=0}^{k-1} \log \left|\mathcal{A}_{j}\right| .
$$

In this paper, we assume that each agent directly transmits the quantized version of its action to other agents. That is, the communication between agents happens over a fully connected graph. However, the fully connected topology can be replaced with a star communication topology without affecting our results. In a star communication topology, each agent transmits the quantized version of its action to a central node, and the central node broadcasts the action of each agent to other agents. Pictorial examples of star and fully connected topologies are shown in Fig. 1. In the next section, we study the asymptotic and transient performance of this algorithm.

Remark 1: The game is non-cooperative in the sense that the agents take individual actions and have individual utility functions. However, we assume that the agents are honest and wish to approach the true NE of the game. That is, despite being self-interested, each agent reports the quantized version of its true action to the others, and does not attempt to deviate from the NE of the game by manipulating its actions.

Remark 2: Note that, in certain applications, agents can directly measure their current utility or gradient values. For example, there are games with utility functions that allow implicit aggregative information exchange between players, e.g. wireless communication (local signal-to-noise-plusinterference-ratio measurements) or smart grid (local voltage/current measurements). In these applications, the NE can be obtained without explicit inter-agent communications [26][29].

In the set-up of this paper, the agents do not have access to such measurements, and explicitly communicate their action values to one another, e.g. because they are not collocated. We model the inter-agent communication channels as digital, which entails quantizing the actions into discrete-valued symbols that may generally be represented as bits. In order to respect the finite capacity of practical channels, the average number of bits transmitted per unit time in our formulation is bounded. This limitation will necessarily degrade the achievable performance. Our aim in this paper is to study the 
impact of this limitation on the performance of Nash-seeking algorithms.

\section{RESUlTS AND Discussions}

In this section, we study three performance measures for gradient-based equilibrium-seeking (ES) algorithms under quantization (2) : $(i)$ the asymptotic rate of exponential meansquare convergence to the Nash equilibrium (NE), $(i i)$ the expected time for agent actions to settle inside a specified neighborhood of the NE, under uniform quantization, and ( iii) the probability that agent actions at a given time $k$ lie outside this neighborhood, also under uniform quantization. The first criterion measures the long-term performance of the system, whereas the other two criteria characterize its transient performance. We assume that $\boldsymbol{x}_{0}$, the vector of initial agent actions, is drawn randomly according to a probability distribution on $\mathbb{R}^{M}$. This will allow us to apply stochastic methods to analyze performance, under mild assumptions on the initial distribution.

\section{A. Lower Bound on Asymptotic Mean Square Convergence Rate}

In this subsection, we find a universal lower bound on the asymptotic convergence rate of any quantized ES scheme of the form (2). Our proof uses the information-theoretic notion of differential entropy power, which has been previously applied to study control with communication constraints; see e.g. [23] and [24]. We assume throughout this subsection that

- the joint probability density function (pdf) $p_{\boldsymbol{x}_{0}}$ of initial actions has finite differential entropy, i.e. $\left|-\int p_{\boldsymbol{x}_{0}}\left(\boldsymbol{x}_{0}\right) \log \left(p_{\boldsymbol{x}_{0}}\left(\boldsymbol{x}_{0}\right)\right) d \boldsymbol{x}_{0}\right|<\infty$.

- the second partial derivatives of the utility functions are bounded above and below as

$$
c_{i} \leq \frac{\partial^{2}}{\partial x^{i^{2}}} U_{i}\left(x^{i}, \boldsymbol{x}^{-i}\right) \leq b_{i}<0, \quad \forall \boldsymbol{x} \in \mathbb{R}^{M}
$$

- the step sizes $\mu_{k}>0$ converge to $\mu^{\star}>0$ as $k \rightarrow \infty$ and also satisfy $\sup _{k \in \mathbb{N}_{0}} \mu_{k}<\frac{1}{\max _{i}\left|c_{i}\right|}$, where $c_{i}$ is the lower bound (4) on the second derivative of the $i$-th agent's utility function with respect to its action.

Next we define equilibrium achieving (EA) quantization schemes.

Definition 1: A quantization scheme $\mathcal{D}$ is equilibriumachieving if all quantized and unquantized actions converge to the NE with time for any initial condition in the support of $p_{\boldsymbol{x}_{0}}$, i.e.

$$
\begin{aligned}
& \lim _{k \longrightarrow \infty} \boldsymbol{x}_{k}=\boldsymbol{x}_{\mathrm{NE}}, \\
& \lim _{k \longrightarrow \infty} \mathcal{D}_{k}\left(\boldsymbol{x}_{k}\right)=\boldsymbol{x}_{\mathrm{NE}} .
\end{aligned}
$$

Remark 3: For the class of equilibrium achieving quantization schemes, the condition (4) only needs to hold in a compact neighborhood of the NE.

Remark 4: Given the first condition in (5), the second can be rewritten as $\lim _{k \rightarrow \infty} \mathcal{D}_{k}\left(x_{k}\right)=\lim _{k \rightarrow \infty} x_{k}$, which expresses the property that the quantized state broadcast by the agents become increasingly refined as time grows. If the second condition does not hold, e.g. if the quantizers are static, we conjecture that asymptotic convergence to the $\mathrm{NE}$ is not generally possible. If the quantizer resolutions are fixed but their bias points can be dynamically adjusted according to previous quantized symbols, then it may be possible to still achieve asymptotic convergence to the NE, using ideas similar to those in [25]. However, in this paper we focus exclusively on quantizers that are (possibly time-varying) functions of the current actions, as in (2).

Next, we define the notion of error decay exponent for the ES algorithm under an EA quantization scheme.

Definition 2: For a given equilibrium-achieving quantization scheme $\mathcal{D}$, let $\epsilon_{k}$ be the difference between agent actions and the NE at time $k$, i.e., $\boldsymbol{\epsilon}_{k}=\boldsymbol{x}_{k}-\boldsymbol{x}_{\mathrm{NE}}$. Then the error decay exponent $(E D E)$ is defined as

$$
\liminf _{k \longrightarrow \infty} \frac{1}{k} \log \mathrm{E}\left[\left\|\boldsymbol{\epsilon}_{k}\right\|_{2}^{2}\right] .
$$

The EDE gives the speed of exponential mean-square convergence of the agents' actions to NE under $\mathcal{D}$, where the expectation is taken with respect to the initial distribution of actions. A more negative exponent indicates faster convergence. We now state the first main result of this paper.

Theorem 1: Let $\mathcal{D}$ be any equilibrium-achieving quantization scheme with average aggregate data rate $R_{\mathcal{D}}$ (3). Then, the error decay exponent is lower-bounded as

$$
\begin{aligned}
\liminf _{k \rightarrow \infty} \frac{1}{k} & \log \mathrm{E}\left[\left\|\epsilon_{k}\right\|_{2}^{2}\right] \\
& \geq \frac{2}{M}\left(\sum_{i=1}^{M} \log \left(1+\left.\mu^{\star} \frac{\partial^{2} U_{i}}{\partial x^{i^{2}}}\right|_{\boldsymbol{x}_{\mathrm{NE}}}\right)-R_{\mathcal{D}}\right) .
\end{aligned}
$$

Proof: Please see Appendix A.

Theorem 1 establishes a universal lower bound on the rate of exponential mean-square convergence that holds for any EA quantization scheme. This lower bound depends on the average aggregate date-rate $R_{\mathcal{D}}$, the second derivatives of the utility functions at the NE, and the number of agents. Recall that a more negative EDE corresponds to faster convergence.

Based on (6), the lower bound decreases (linearly) as $R_{\mathcal{D}}$ increases. This reflects the fact that each agent has more accurate information about the actions of the others and hence can make better decisions. Furthermore, the bound increases with the second derivatives of the utility functions. This is because a less negative second derivative indicates a flatter utility function, hence slower convergence to the NE.

Though the bound above may be conservative, unlike previous work it does not impose any particular structure on the quantization scheme, and delineates a universal trade-off between convergence rate, utility functions, data rate, and the number of agents.

\section{B. Transient Performance}

In this subsection, we investigate the transient behavior of the equilibrium-seeking (ES) algorithm (2) under a uniform, time-invariant quantization scheme. We make the following assumptions on the game $\mathcal{G}$ and the ES algorithm (1): 
- The NE of the game $\mathcal{G}$ belongs to the open, bounded and connected set $\mathcal{R} \subset \mathbb{R}^{M}$ which has non-zero Lebesgue measure.

- $\boldsymbol{x}_{0}$, i.e, the initial action of agents, is randomly drawn from $\mathcal{R}$.

- $\frac{\partial}{\partial x^{i}} U_{i}\left(x^{i}, \boldsymbol{x}^{-i}\right)$ is twice continuously differentiable for all $i$.

- The ES algorithm under perfect communication, i.e., the update rule (1), is a pseudo-contraction mapping. That is

$$
\left\|\boldsymbol{x}_{k}-\boldsymbol{x}_{\mathrm{NE}}\right\|_{2} \leq \alpha\left\|\boldsymbol{x}_{k-1}-\boldsymbol{x}_{\mathrm{NE}}\right\|_{2},
$$

where $\alpha \in[0,1)$ and

$$
x_{k}^{i}=x_{k-1}^{i}+\mu_{k-1} \frac{\partial}{\partial x^{i}} U_{i}\left(x_{k-1}^{i}, \boldsymbol{x}_{k-1}^{-i}\right) .
$$

- The sequence $\left\{\mu_{k}\right\}_{k=0}^{\infty}$ is assumed to be bounded.

Let $d$ be the diameter of $\mathcal{R}$, that is, $d=$ $\sup \left\{\|\boldsymbol{x}-\boldsymbol{y}\|_{2}: \boldsymbol{x}, \boldsymbol{y} \in \mathcal{R}\right\}$. Let $\mathrm{B}\left(\boldsymbol{x}_{c}, d / 2\right)$ be the smallest ball containing $\mathcal{R}$ where $\mathrm{B}\left(\boldsymbol{x}_{c}, d / 2\right)$ represents a closed ball in Euclidean norm centered at $\boldsymbol{x}_{c}$ with radius $d / 2$. Let $\mathrm{Q}\left(\boldsymbol{x}_{c}, 3 d / 2\right)$ be the cube centered at $\boldsymbol{x}_{c}$ with side length $3 d$. In this subsection, we assume that agents employ a uniform, time-invariant quantization scheme denoted by $\mathcal{D}_{\mathrm{u}}$. Under $\mathcal{D}_{\mathrm{u}}$, the intersection of $\mathrm{Q}\left(\boldsymbol{x}_{c}, 3 d / 2\right)$ and action space of each agents is uniformly quantized with the quantization step $\delta$. The ES update rule under the uniform quantization scheme $\mathcal{D}_{\mathrm{u}}$ is given by

$$
x_{k+1}^{i}=x_{k}^{i}+\mu_{k} \frac{\partial}{\partial x^{i}} U_{i}\left(x_{k}^{i}, \mathcal{D}_{\mathrm{u}}\left(\boldsymbol{x}_{k}^{-i}\right)\right), \forall i .
$$

Since the quantization scheme $\mathcal{D}_{\mathrm{u}}$ is only defined on $\mathrm{Q}\left(\boldsymbol{x}_{c}, 3 d / 2\right)$, we need to ensure that the actions of agents will always stay in $\mathrm{Q}\left(\boldsymbol{x}_{c}, 3 d / 2\right)$. In Appendix $\mathrm{C}$, it is shown that if $\boldsymbol{x}_{0}$, the initial action of agents, belongs to $\mathcal{R}$ and the quantization step $\delta$ is sufficiently small, then, the actions of agents will always stay in $\mathrm{Q}\left(\boldsymbol{x}_{c}, 3 d / 2\right)$. A sufficient condition for $\delta$ is given by

$$
\begin{aligned}
\sup _{k} \mu_{k} & \left(\delta \sqrt{\sum_{i} \Phi_{i}^{2}}+\frac{1}{2} \delta^{2}\left(M \sqrt{\sum_{i} \Psi_{i}^{2}}+\sqrt{\sum_{i} \eta_{i}^{2}}\right)\right) \\
\leq(1-\alpha) d, &
\end{aligned}
$$

where $\Phi_{i}, \Psi_{i}$ and $\eta_{i}$ are given by

$$
\begin{aligned}
\Phi_{i} & =\sup _{\boldsymbol{x} \in \mathrm{Q}\left(\boldsymbol{x}_{c}, 3 d / 2\right)} \sum_{j \neq i}\left|\frac{\partial^{2}}{\partial^{2} x^{j} x^{i}} U_{i}\left(x^{i}, \boldsymbol{x}^{-i}\right)\right|, \\
\Psi_{i} & =\sup _{\boldsymbol{x} \in \mathrm{Q}\left(\boldsymbol{x}_{c}, 3 d / 2\right)}\left\|\nabla^{2} \frac{\partial}{\partial x^{i}} U_{i}\left(x^{i}, \boldsymbol{x}^{-i}\right)\right\|_{2}, \\
\eta_{i} & =\sup _{\boldsymbol{x} \in \mathrm{Q}\left(\boldsymbol{x}_{c}, 3 d / 2\right)}\left|\frac{\partial^{3}}{\partial x^{i^{3}}} U_{i}\left(x^{i}, \boldsymbol{x}^{-i}\right)\right| .
\end{aligned}
$$

respectively, where $\nabla^{2}(\cdot)$ is the Hessian operator (see Lemma 3 in Appendix $\mathrm{C}$ and its proof for more details). The left hand side of (8) is an upper bound on the distortion induced by the quantization scheme $\mathcal{D}_{\mathrm{u}}$. Thus, (8) essentially implies that the quantization scheme $\mathcal{D}_{\mathrm{u}}$ is well defined if the distortion caused by the quantization scheme at each time step is small enough.

The term $\Phi_{i}$ represents the sensitivity of update rule of the $i$-th agent to the actions of other agents. When agents are less sensitive to each other's actions, according to (8), a relatively large quantization step $\delta$ can be chosen without introducing a large amount of distortion in the evolution of the ES algorithm. However, when agents are highly sensitive to each other's actions, a high resolution quantization scheme should be employed to avoid a large amount of distortion. Also, according to (8), small values of the step size result in small distortion values. Since each agent modifies its action by adding the term $\mu_{k} \frac{\partial}{\partial x^{i}} U_{i}\left(x_{k}^{i}, \mathcal{D}_{\mathbf{u}}\left(\boldsymbol{x}_{k}^{-i}\right)\right)$ to its previous action, a small value of step size results in a small value of distortion at the cost of a slow convergence speed.

We use $\mathrm{E}[\mathcal{N}]$ to denote the expected time required for $\boldsymbol{x}_{k}$ to settle inside $\mathrm{B}\left(\boldsymbol{x}_{\mathrm{NE}}, r\right)$. A small value of $\mathrm{E}[\mathcal{N}]$ indicates that the ES algorithm, on average, quickly approaches the NE whereas a large value of the $E[\mathcal{N}]$ indicates a relatively slow convergence. Due to the quantization distortion, the radius of $\mathrm{B}\left(\boldsymbol{x}_{\mathrm{NE}}, r\right)$ cannot be arbitrarily small. If $r$ is less than the total quantization distortion, one cannot guarantee that agents' actions will eventually settle inside $\mathrm{B}\left(\boldsymbol{x}_{\mathrm{NE}}, r\right)$ as $k$ becomes large. Here, we assume that $r>\theta$ where $\theta$ is given by

$\theta=\frac{\sup _{k} \mu_{k}}{1-\alpha}\left(\delta \sqrt{\sum_{i} \Phi_{i}^{2}}+\frac{1}{2} \delta^{2}\left(M \sqrt{\sum_{i} \Psi_{i}^{2}}+\sqrt{\sum_{i} \eta_{i}^{2}}\right)\right)$.

Here, $\theta$ represents an upper bound on the aggregate distortion caused by the quantization scheme $\mathcal{D}_{\mathrm{u}}$ over time (see Appendix $\mathrm{C}$ for more details). The next theorem provides an upper bound on $\mathrm{E}[\mathcal{N}]$.

Theorem 2: Consider the uniform quantization scheme $\mathcal{D}_{\mathrm{u}}$ with the quantization step $\delta$ satisfying (8). Let $E[\mathcal{N}]$ denote the expected time required for the ES algorithm under $\mathcal{D}_{\mathrm{u}}$ to settle in $\mathrm{B}\left(\boldsymbol{x}_{\mathrm{NE}}, r\right)$ with $r>\theta$. Then, $\mathrm{E}[\mathcal{N}]$ is upper bounded as

$\mathrm{E}[\mathcal{N}] \leq$

$$
\frac{1}{\log \left(\frac{1}{\alpha}\right)}\left(\mathrm{E}\left[\log \left(\frac{\left\|\boldsymbol{x}_{0}-\boldsymbol{x}_{\mathrm{NE}}\right\|_{2}}{r-\theta}\right) 1\left\{\frac{\left\|\boldsymbol{x}_{0}-\boldsymbol{x}_{\mathrm{NE}}\right\|_{2}}{r-\theta}>1\right\}\right]\right.
$$

Proof: Please see Appendix C.

Theorem 2 provides an upper bound on the expected time required for the actions of agents to settle inside a ball of radius $r$ centered at the NE. This upper bound is controlled by $\alpha, \theta, r$ and the distribution of the initial actions of agents. According to this theorem, the effect of $\alpha$ on the expected time is manifested through the multiplicative factor $\frac{1}{\log \left(\frac{1}{\alpha}\right)}$ with $\alpha \in[0,1)$. As $\alpha$ becomes closer to zero, the distance between the actions of agents and the NE decays faster due to the pseudo-contraction property of the non-quantized update rule (see equation (28) in Appendix C for more details). Thus, the average time required to settle inside $\mathrm{B}\left(\boldsymbol{x}_{\mathrm{NE}}, r\right)$ becomes smaller as $\alpha$ decreases.

Note $\mathrm{E}[\mathcal{N}]$ is a non-increasing function of $r$. That is, as $r$ becomes small, it takes more time for the ES algorithm (7) to settle in $\mathrm{B}\left(\boldsymbol{x}_{\mathrm{NE}}, r\right)$. This observation is also consistent with 
our result in Theorem 2, i.e., the upper bound on the $E[\mathcal{N}]$ increases as $r$ becomes small. Finally, Theorem 2 suggests that the expected time required to settle inside $\mathrm{B}\left(\boldsymbol{x}_{\mathrm{NE}}, r\right)$ is influenced by the distribution of initial actions of agents, $p_{\boldsymbol{x}_{0}}(\boldsymbol{x})$. Observe that, when $p_{\boldsymbol{x}_{0}}(\boldsymbol{x})$ is highly concentrated around the Nash equilibrium, the ES algorithm (7) requires less time to settle inside $\mathrm{B}\left(\boldsymbol{x}_{\mathrm{NE}}, r\right)$ compared to the case that $p_{\boldsymbol{x}_{0}}(\boldsymbol{x})$ has a low degree of concentration around the NE.

The upper bound in Theorem 2 depends on the distance between the initial action of agents and the NE, i.e., $\left\|\boldsymbol{x}_{0}-\boldsymbol{x}_{\mathrm{NE}}\right\|_{2}$. Since both $\boldsymbol{x}_{0}$ and $\boldsymbol{x}_{\mathrm{NE}}$ belong to $\mathcal{R}$, one can use the fact that $\left\|\boldsymbol{x}_{0}-\boldsymbol{x}_{\mathrm{NE}}\right\|_{2} \leq d$ to obtain an upper bound on the $\mathrm{E}[\mathcal{N}]$ which is independent of $\boldsymbol{x}_{0}$ and $\boldsymbol{x}_{\mathrm{NE}}$. We state this result in the next corollary.

Corollary 1: The expected time required for the ES algorithm under $\mathcal{D}_{\mathrm{u}}$ to settle in $\mathrm{B}\left(\boldsymbol{x}_{\mathrm{NE}}, r\right)$ can be upper bounded as

$$
\mathrm{E}[\mathcal{N}] \leq \frac{1}{\log \left(\frac{1}{\alpha}\right)} \log \left(\frac{d}{r-\theta}\right)
$$

Next, we turn our attention to the other performance measure for the transient behavior of the ES algorithm (7) under the uniform quantization scheme $\mathcal{D}_{\mathrm{u}}$. Here, we are interested in the probability that $\boldsymbol{x}_{k}$ lies outside a ball of radius $r>\theta$ around the NE, i.e.,

$$
\operatorname{Pr}\left\{\left\|\boldsymbol{x}_{k}-\boldsymbol{x}_{\mathrm{NE}}\right\|_{2}>r\right\} .
$$

Note that $\operatorname{Pr}\left\{\left\|\boldsymbol{x}_{k}-\boldsymbol{x}_{\mathrm{NE}}\right\|_{2}>r\right\}$ is a function of $k$, and decays to zero as $k$ tends to infinity. For a given $k$, a small value of $\operatorname{Pr}\left\{\left\|\boldsymbol{x}_{k}-\boldsymbol{x}_{\mathrm{NE}}\right\|_{2}>r\right\}$ indicates that $\boldsymbol{x}_{k}$ approaches the NE at a higher speed compared to a large value of $\operatorname{Pr}\left\{\left\|\boldsymbol{x}_{k}-\boldsymbol{x}_{\mathrm{NE}}\right\|_{2}>r\right\}$. The next theorem provides an upper bound on the probability that $\boldsymbol{x}_{k}$ lies outside B $\left(\boldsymbol{x}_{\mathrm{NE}}, r\right)$.

Theorem 3: Consider the uniform quantization scheme $\mathcal{D}_{\mathrm{u}}$ with the quantization step $\delta$ satisfying (8). Then, the probability that $\boldsymbol{x}_{k}$ lies outside $\mathrm{B}\left(\boldsymbol{x}_{\mathrm{NE}}, r\right)$ with $r>\theta$ is upper bounded as

$\operatorname{Pr}\left\{\left\|\boldsymbol{x}_{k}-\boldsymbol{x}_{\mathrm{NE}}\right\|_{2}>r\right\} \leq \min \left(1, \mathrm{e}^{-\frac{r-\theta}{\alpha^{k}}} \mathrm{E}\left[\mathrm{e}^{\left\|\boldsymbol{x}_{0}-\boldsymbol{x}_{\mathrm{NE}}\right\|_{2}}\right]\right)$

Theorem 3 provides an upper bound on the probability that $\boldsymbol{x}_{k}$ lies outside of the ball radius $r$ around the NE at a given time. According to Theorem 3, this probability decays to zero at least double exponentially with $k$. Also, the decay rate of this probability depends on the contraction constant $\alpha$. As $\alpha$ becomes small, the distance between agents' actions and the NE decays faster. Hence, the probability that $\boldsymbol{x}_{k}$ lies outside $\mathrm{B}\left(\boldsymbol{x}_{\mathrm{NE}}, r\right)$ decays faster to zero, and $\boldsymbol{x}_{k}$ with high probability lies inside $\mathrm{B}\left(\boldsymbol{x}_{\mathrm{NE}}, r\right)$. The term $\mathrm{E}\left[\mathrm{e}^{\left\|\boldsymbol{x}_{0}-\boldsymbol{x}_{\mathrm{NE}}\right\|_{2}}\right]$ in (9) indicates the effect of the distribution of $\boldsymbol{x}_{0}$ on $\operatorname{Pr}\left\{\left\|\boldsymbol{x}_{k}-\boldsymbol{x}_{\mathrm{NE}}\right\|_{2}>r\right\}$. That is, when the distribution of $\boldsymbol{x}_{0}$ is more concentrated around the NE, we expect $\boldsymbol{x}_{k}$ to approach the NE at a faster speed. Note that for a given $k, \operatorname{Pr}\left\{\left\|\boldsymbol{x}_{k}-\boldsymbol{x}_{\mathrm{NE}}\right\|_{2}>r\right\}$ is a non-increasing function of $r$. That is, $\boldsymbol{x}_{k}$ lies outside $\mathrm{B}\left(\boldsymbol{x}_{\mathrm{NE}}, r\right)$ with high probability as $r$ becomes small. This behavior is consistent with the upper bound in Theorem 3 .

One can use the fact that $\left\|\boldsymbol{x}_{0}-\boldsymbol{x}_{\mathrm{NE}}\right\|_{2} \leq d$ to obtain an upper bound on $\operatorname{Pr}\left\{\left\|\boldsymbol{x}_{k}-\boldsymbol{x}_{\mathrm{NE}}\right\|_{2}>r\right\}$ which is independent of $\boldsymbol{x}_{0}$ and $\boldsymbol{x}_{\mathrm{NE}}$. This result is stated in the next corollary.

Corollary 2: The probability that $\boldsymbol{x}_{k}$ lies outside B $\left(\boldsymbol{x}_{\mathrm{NE}}, r\right)$ can be upper bounded as

$$
\operatorname{Pr}\left\{\left\|\boldsymbol{x}_{k}-\boldsymbol{x}_{\mathrm{NE}}\right\|_{2}>r\right\} \leq \min \left(1, \mathrm{e}^{d-\frac{r-\theta}{\alpha^{k}}}\right) .
$$

Finally, the upper bound in Corollary 2 can be used to obtain an upper bound on the number of time-steps required for $\boldsymbol{x}_{k}$ to lie in B $\left(\boldsymbol{x}_{\mathrm{NE}}, r\right)$ with a given certainty level. Let $N_{p}$ be the number of time-steps required for $\boldsymbol{x}_{k}$ to lie in B $\left(\boldsymbol{x}_{\mathrm{NE}}, r\right)$ with the probability at least equal to $p$. Then, using (10), $N_{p}$ can be upper bounded by the smallest positive integer satisfying

$$
d-(r-\theta) \leq \alpha^{k} \log (1-p) .
$$

Theorem 3 and Corollary 2 can be used to obtain bounds on the required number of quantization levels to guarantee that the agents' actions at time $k$ lie inside a ball of radius $r$ around the NE with a given probability. Also, Theorem 2 and Corollary 1 give rise to bounds on the required number of quantization levels to guarantee an average settling time. Finally, Theorem 1 provides guidelines on the required average aggregate data rate for achieving a desired speed of exponential convergence to the NE.

\section{An Adaptive EA Quantization Scheme}

In this subsection, based on our analysis of the uniform quantization scheme $\mathcal{D}_{\mathrm{u}}$, we propose an adaptive EA quantization scheme under which the ES algorithm converges to the NE. We refer to this quantization scheme as $\mathcal{D}_{\mathrm{a}}$. Later, in the numerical result section, we study the error decay exponent of $\mathcal{D}_{\mathrm{a}}$. Recall that, the NE belongs to the region $\mathcal{R}$ with the diameter $d$. The basic idea behind the adaptive quantization scheme $\mathcal{D}_{\mathrm{a}}$ is to reduce the size of the known region around the NE in each time-step.

Let $\mathcal{R}_{k}$ denote the region which the NE belongs to at time $k$ under the quantization scheme $\mathcal{D}_{\mathrm{a}}$. The quantization scheme $\mathcal{D}_{\mathrm{a}}$ is designed such that the diameter of $\mathcal{R}_{k}$ converges to (9) zero as $k$ tends to infinity. Under the quantization scheme $\mathcal{D}_{\text {a }}$, initially, the intersection of action space of each agent with $\mathrm{Q}\left(\boldsymbol{x}_{c}, 3 d / 2\right)$ is quantized with the quantization step $\delta_{0}$ which satisfies the following inequality

$$
\begin{aligned}
\sup _{k} \mu_{k} & \left(\delta_{0} \sqrt{\sum_{i} \Phi_{i}^{2}}+\frac{1}{2} \delta_{0}^{2}\left(M \sqrt{\sum_{i} \Psi_{i}^{2}}+\sqrt{\sum_{i} \eta_{i}^{2}}\right)\right) \\
\leq \hat{\alpha} d &
\end{aligned}
$$

where $\hat{\alpha}$ is a constant arbitrarily selected from the interval $(0,1-\alpha)$. Using equation (28) in Appendix $\mathrm{C}$, the distance between $\boldsymbol{x}_{1}$ and the NE under $\mathcal{D}_{\mathrm{a}}$ can be upper bounded as

$$
\begin{gathered}
\left\|\boldsymbol{x}_{1}-\boldsymbol{x}_{\mathrm{NE}}\right\|_{2} \leq \alpha\left\|\boldsymbol{x}_{0}-\boldsymbol{x}_{\mathrm{NE}}\right\|_{2} \\
+\sup _{k} \mu_{k}\left(\delta_{0} \sqrt{\sum_{i} \Phi_{i}^{2}}+\frac{1}{2} \delta_{0}^{2}\left(M \sqrt{\sum_{i} \Psi_{i}^{2}}+\sqrt{\sum_{i} \eta_{i}^{2}}\right)\right), \\
\leq(\alpha+\hat{\alpha}) d,
\end{gathered}
$$

which implies that the NE belongs to the ball of radius $(\alpha+\hat{\alpha}) d$ around $\boldsymbol{x}_{1}$. In the second time-step, 
$\mathrm{Q}\left(\boldsymbol{x}_{1},(\alpha+\hat{\alpha}) d\right) \cap \mathrm{Q}\left(\boldsymbol{x}_{c}, 3 d / 2\right)$ is considered as $\mathcal{R}_{1}$ and the intersection of each agent's action space with the $\mathcal{R}_{1}$ is quantized. Similarly, at time $k, k \geq 1$, we have $\mathcal{R}_{k}=$ $\mathrm{Q}\left(\boldsymbol{x}_{k}, d_{k}\right) \cap \mathrm{Q}\left(\boldsymbol{x}_{c}, 3 d / 2\right)$ where $d_{i}=(\alpha+\hat{\alpha}) d_{i-1}$ with $d_{0}=d$. Then, the intersection of action space of each agent with $\mathcal{R}_{k}$ is quantized. Also, the quantization step at time $k$, $\delta_{k}$, is chosen such that the following inequality is satisfied

$$
\begin{aligned}
\sup _{k} \mu_{k} & \left(\delta_{k} \sqrt{\sum_{i} \Phi_{i}^{2}}+\frac{1}{2} \delta_{k}^{2}\left(M \sqrt{\sum_{i} \Psi_{i}^{2}}+\sqrt{\sum_{i} \eta_{i}^{2}}\right)\right) \\
\leq \hat{\alpha} d_{k}, &
\end{aligned}
$$

Since $d_{k}$ converges to zero as $k$ tends to infinity, the actions of agents and their quantized versions, under the quantization scheme $\mathcal{D}_{\text {a }}$, converge to the NE as $k$ tends to infinity which implies that $\mathcal{D}_{\mathrm{a}}$ is an EA quantization scheme. Algorithm 1 shows the different steps of the adaptive quantization scheme $\mathcal{D}_{\mathrm{a}}$.

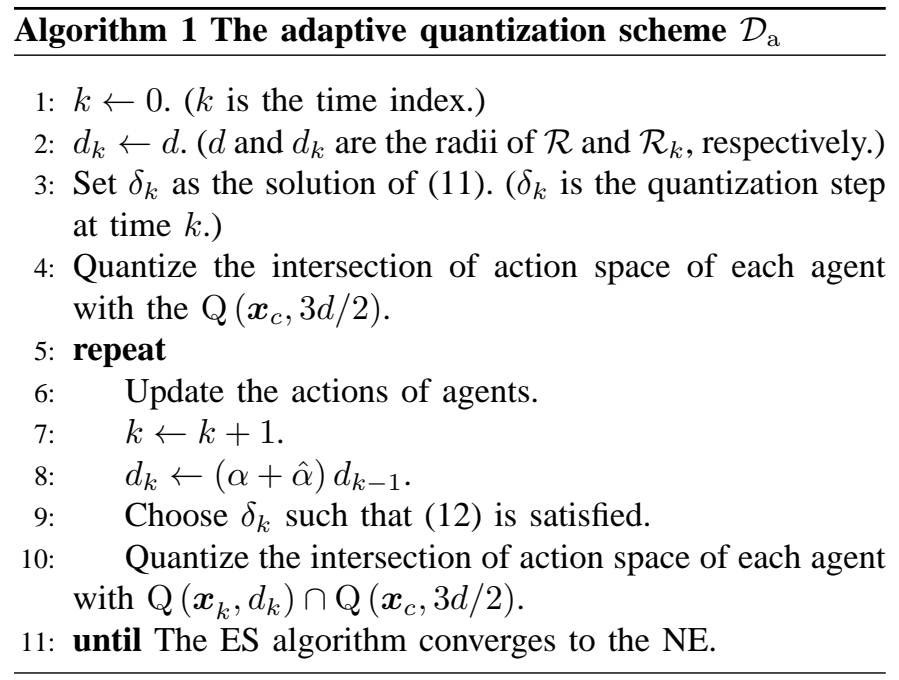

\section{NumericAl RESUlts}

In this section, we present our numerical results for a noncooperative game with 5 agents seeking to maximize their utility functions. The utility function of $i$-th agent is given by

$$
U_{i}\left(x^{i}, \boldsymbol{x}^{-i}\right)=\frac{t_{i i}}{2}\left(x^{i}\right)^{2}+x^{i}\left(\sum_{j \neq i} t_{i j} x^{j}-l_{i}\right),
$$

where $t_{i i}<0$ for all $i$ and $t_{i j}, l_{i} \in \mathbb{R}$. Utility functions of the form (13) arise in many engineering applications such as analyzing the bidding behavior of a group of generators competing for maximizing their profits in an electricity market, e.g., see [22]-[21]. Let $T$ be an $M$-by- $M$ matrix with the $(i, j)$-th entry equal to $t_{i j}$. We assume that $T$ is negative definite. Since $T$ is invertible, it can be easily verified, using the Karush-Kuhn-Tucker conditions [30], that the quadratic game with the utility functions (13) admits a unique Nash equilibrium. For this quadratic game, the ES algorithm under perfect communication condition can be written as

$$
\boldsymbol{x}_{k+1}=(I+\mu T) \boldsymbol{x}_{k}-\mu \boldsymbol{l}
$$

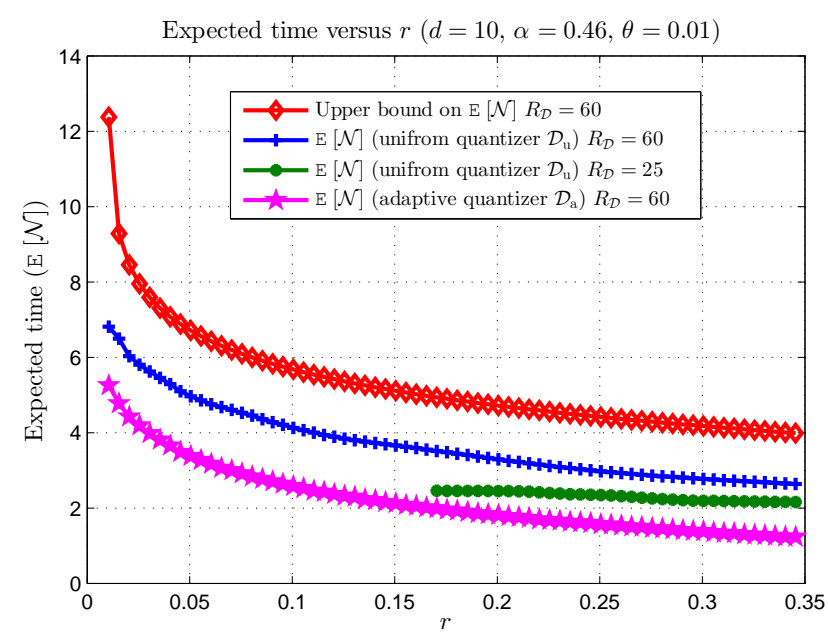

Fig. 2. The expected time required for the ES algorithm to settle in a ball of radius $r$ around the NE for the fixed and adaptive quantizers as a function of $r$

where $\boldsymbol{l}=\left[l_{1}, \cdots, l_{M}\right]^{\top}$. The step size $\mu$ is selected such that the spectral radius of $I+\mu T$ is strictly less than one. In our numerical results, we assume that the NE belongs to a hypercube $\mathcal{R}$ whose side length is equal to $\frac{10}{\sqrt{5}}$. Also, the vector of initial actions of agents, $\boldsymbol{x}_{0}$, is assumed to be uniformly distributed on $\mathcal{R}$.

Fig. 2 illustrates the expected time required for $\boldsymbol{x}_{k}$ to settle inside $\mathrm{B}\left(\boldsymbol{x}_{\mathrm{NE}}, r\right)$ as a function of $r$ for different quantization schemes and different values of average aggregate data rates $R_{\mathcal{D}}$. In this figure, $\theta, d$ and $\alpha$ are set to $10^{-2}, 10$ and 0.46 , respectively. As $r$ becomes large, the ES algorithm under both $\mathcal{D}_{\mathrm{u}}$ and $\mathcal{D}_{\mathrm{a}}$ requires less time to settle inside $\mathrm{B}\left(\boldsymbol{x}_{\mathrm{NE}}, r\right)$, and as a result, the expected time, under both $\mathcal{D}_{\mathrm{u}}$ and $\mathcal{D}_{\mathrm{a}}$, decreases as $r$ becomes large. As shown in Fig. 2, the expected time under the fixed quantization scheme $\mathcal{D}_{\mathrm{u}}$ is limited by the upper bound provided by Theorem 2. According Fig. 2, the ES algorithm under the EA quantization scheme $\mathcal{D}_{\text {a }}$, on average, requires less time to settle inside $\mathrm{B}\left(\boldsymbol{x}_{\mathrm{NE}}, r\right)$ compared to the fixed quantization scheme $\mathcal{D}_{\mathrm{u}}$. The fast convergence of the ES algorithm under $\mathcal{D}_{\mathrm{a}}$ is due to the flexible structure of the EA quantization scheme $\mathcal{D}_{\mathrm{a}}$.

In Fig. 2, the ES algorithm under $\mathcal{D}_{\mathrm{u}}$ with coarse quantization scheme $R_{\mathcal{D}}=25$ cannot settle inside the ball $\mathrm{B}\left(\boldsymbol{x}_{\mathrm{NE}}, r\right)$ when $r$ is small which is due to the large amount of distortion caused by the coarse quantization scheme. However, as $r$ becomes large, the ES algorithm under the coarse quantization scheme settles inside the ball $\mathrm{B}\left(\boldsymbol{x}_{\mathrm{NE}}, r\right)$ faster than the fine quantization scheme $R_{\mathcal{D}}=60$. Note that any quantization scheme introduces an extra displacement to the agents' action at each time-step. A coarse quantization scheme causes a bigger displacement, compared to a fine quantization scheme, which results in a lower expected time to settle inside $\mathrm{B}\left(\boldsymbol{x}_{\mathrm{NE}}, r\right)$ (when $r$ is large enough) .

Fig. 3 illustrates the probability that the actions of agents lie outside $\mathrm{B}\left(\boldsymbol{x}_{\mathrm{NE}}, r\right)$ at time $k$, i.e., $\operatorname{Pr}\left\{\left\|\boldsymbol{x}_{k}-\boldsymbol{x}_{\mathrm{NE}}\right\|_{2}>r\right\}$, 


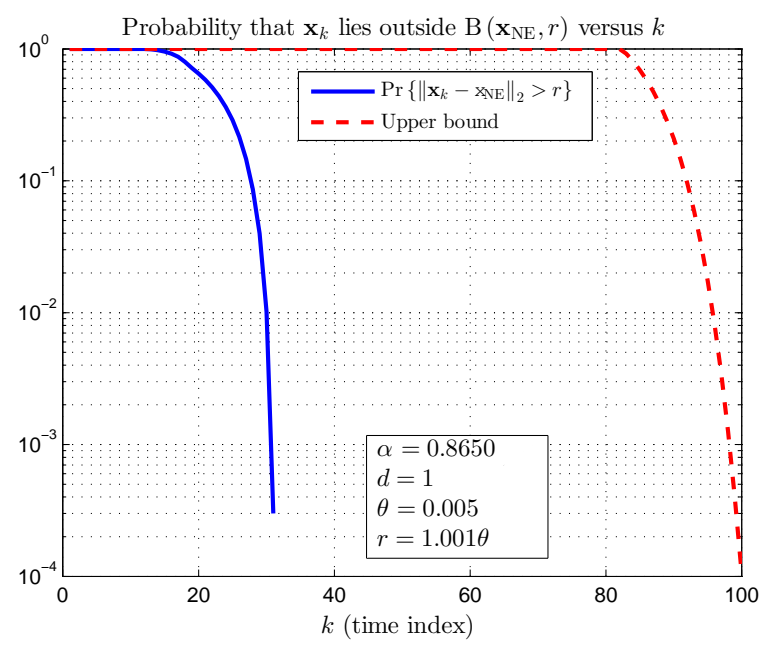

Fig. 3. The probability that the actions of agents at time $k$ lie outside the ball of radius $r$ around the NE and its upper bound as a function of $k$.

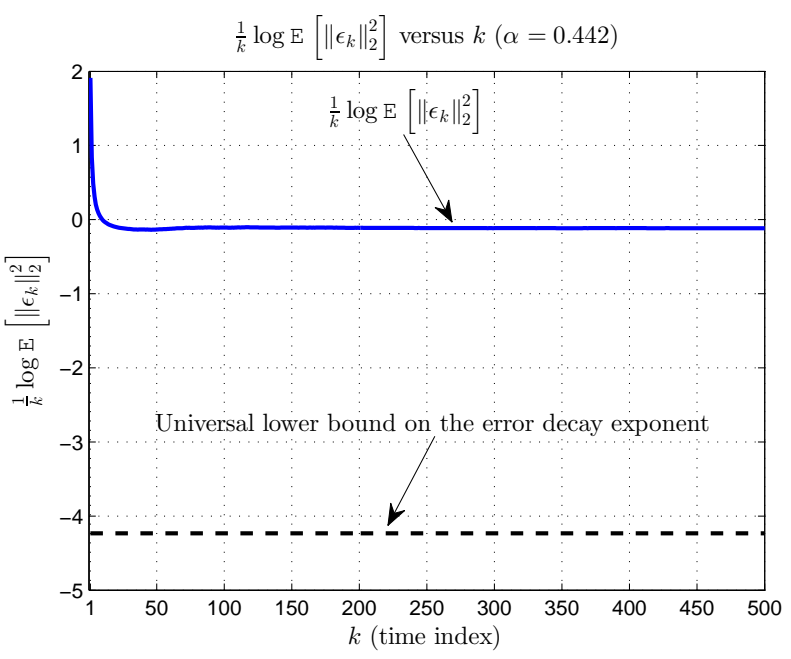

Fig. 4. log-mean-square-error-norm divided by $k$ under the adaptive quantization scheme $\mathcal{D}_{\mathrm{a}}$ as a function of $k$.

and the upper bound on $\operatorname{Pr}\left\{\left\|\boldsymbol{x}_{k}-\boldsymbol{x}_{\mathrm{NE}}\right\|_{2}>r\right\}$ provided by the Theorem 3. In this figure, $\alpha, d$ and $\theta$ are set to $0.8056,1$ and 0.005 , respectively. In Fig. $3, \operatorname{Pr}\left\{\left\|\boldsymbol{x}_{k}-\boldsymbol{x}_{\mathrm{NE}}\right\|_{2}>r\right\}$ is numerically computed by taking average over 100000 samples. Here, we have assumed that each agent will use 12 bits to quantize its action. As Fig. 3 shows, the probability of lying outside $\mathrm{B}\left(\boldsymbol{x}_{\mathrm{NE}}, r\right)$ decays to zero as $k$ becomes large due to the contraction property of the update rule. Also, this probability lies below the double exponentially decaying upper bound, provided by the Theorem 3, as shown in Fig. 3 which indicates that the probability of lying outside $\mathrm{B}\left(\boldsymbol{x}_{\mathrm{NE}}, r\right)$ decays at least double exponentially to zero as the time index $k$ becomes large.

Fig. 4 demonstrates the behavior of log-mean-square-errornorm divided by $k$, i.e., $\frac{1}{k} \log \mathrm{E}\left[\left\|\boldsymbol{\epsilon}_{k}\right\|_{2}^{2}\right]$, under the adaptive quantizer $\mathcal{D}_{\mathrm{a}}$ as a function of the number of time-steps. As Fig. 4 shows $\frac{1}{k} \log \mathrm{E}\left[\left\|\epsilon_{k}\right\|_{2}^{2}\right]$ stays above -4.2318 , the predicted lower bound by the Theorem 1 , as $k$ becomes large.

\section{CONCLUSiON}

In this paper, we have investigated the impact of quantized inter-agent communications on the convergence behavior of the gradient-based Nash equilibrium seeking (ES) algorithm in non-cooperative games. We used the information-theoretic notion of entropy power to establish a universal lower bound on the rate of exponential mean square convergence of such algorithms, assuming equilibrium-achieving quantizers. This lower bound signifies the impact of inter-agent communication data rates on the convergence speed of the ES algorithm to the Nash equilibrium (NE). Next, the transient behavior of the ES algorithm under quantized message passing among agents was examined. To this end, we established an upper bound on the expected time required for the ES algorithm to settle inside a ball centered at the NE under a uniform quantization scheme. We also derive an upper bound on the probability that agents' actions at a given time lie outside a ball around the Nash equilibrium. These two results only concern the behavior of the ES algorithm until it reaches a neighborhood of the Nash equilibrium, and do not make any assumption on the convergence of the ES algorithm. Finally, we proposed an equilibrium achieving quantization scheme which, without decreasing the number of quantization levels, allows the ES algorithm to arrive at the NE through an adaptive refinement of the uncertainty region.

\section{APPENDIX A PROOF OF THEOREM 1}

In this appendix, first the notion of conditional entropy power of a random vector is introduced. Then, based on the entropy power, a lower bound on the error decay exponent is derived.

Let $\boldsymbol{x} \in \mathbb{R}^{M}$ be a random vector with absolutely continuous distribution with respect to the Lebesgue measure on $\mathbb{R}^{M}$. Then, the entropy power of $\boldsymbol{x}$ conditioned on the event $A=a$, denoted by $\operatorname{EP}[\boldsymbol{x} \mid A=a]$, is defined as

$$
\mathrm{EP}[\boldsymbol{x} \mid A=a]=\frac{1}{2 \pi \mathrm{e}} \mathrm{e}^{\frac{2}{M} \mathrm{~h}[\boldsymbol{x} \mid A=a]},
$$

where $A$ is a random variable and $\mathrm{h}[\boldsymbol{x} \mid A=a]$ is the conditional entropy of $\boldsymbol{x}$ given $A=a$ defined as

$\mathrm{h}[\boldsymbol{x} \mid A=a]=-\int \log (p(\boldsymbol{x} \mid A=a)) p(\boldsymbol{x} \mid A=a) d \boldsymbol{x}$,

where $p(\boldsymbol{x} \mid A=a)$ is the conditional distribution of $\boldsymbol{x}$ given $A=a$. Using the entropy maximization property of Gaussian distributions, EP $[\boldsymbol{x} \mid A=a]$ can be upper bounded as [13]

$$
\mathrm{EP}[\boldsymbol{x} \mid A=a] \leq \mathrm{e}^{1 / M-1} \mathrm{E}\left[\|\boldsymbol{x}\|_{2}^{2} \mid A=a\right]
$$

where $\mathrm{E}\left[\|\boldsymbol{x}\|_{2}^{2} \mid A=a\right]$ is conditional expectation of $\|\boldsymbol{x}\|_{2}^{2}$ given $A=a$. Using (14), the average conditional entropy power of $\boldsymbol{x}$ given $A=a$, i.e, $\mathrm{E}_{A}[\mathrm{EP}[\boldsymbol{x} \mid A=a]]$ can be upper bounded as

$$
\mathrm{E}_{A}[\mathrm{EP}[\boldsymbol{x} \mid A]] \leq \mathrm{e}^{1 / M-1} \mathrm{E}\left[\|\boldsymbol{x}\|_{2}^{2}\right] .
$$


In this appendix, we use (15) to obtain a lower bound on the decay exponent of error of the update rule (2) under EA quantization schemes. Using (15), $\mathrm{E}\left[\left\|\epsilon_{k}\right\|_{2}^{2}\right]$ can be lower bounded as

$$
\begin{aligned}
\mathrm{E}\left[\left\|\boldsymbol{\epsilon}_{k}\right\|_{2}^{2}\right] & \geq \mathrm{e}^{1-\frac{1}{M}} \mathrm{E}\left[\mathrm{EP}\left[\boldsymbol{\epsilon}_{k} \mid \mathcal{I}_{k-1}\right]\right] \\
& \stackrel{(*)}{\geq} \frac{\mathrm{e}^{1-\frac{1}{M}}}{2 \pi \mathrm{e}} \mathrm{e}^{\frac{2}{M} \mathrm{E}\left[\mathrm{h}\left[\boldsymbol{\epsilon}_{k} \mid \mathcal{I}_{k-1}\right]\right]}
\end{aligned}
$$

where $\mathcal{I}_{k-1}=\left\{\mathcal{D}_{i}\left(\boldsymbol{x}_{i}\right)=\boldsymbol{a}_{i}\right\}_{i=0}^{k-1}, \boldsymbol{a}_{i} \in \mathcal{A}_{i}$ is a possible output of the quantization scheme $\mathcal{D}$ at time $i$ and $(*)$ follows from the Jensen inequality. To evaluate (16), we need to evaluate $\mathrm{h}\left[\boldsymbol{\epsilon}_{k} \mid \mathcal{I}_{k-1}\right]$ which can be written as

$$
\begin{aligned}
\mathrm{h}\left[\boldsymbol{\epsilon}_{k} \mid \mathcal{I}_{k-1}\right] & =\mathrm{h}\left[\boldsymbol{x}_{k}-\boldsymbol{x}_{\mathrm{NE}} \mid \mathcal{I}_{k-1}\right] \\
& \stackrel{(*)}{=} \mathrm{h}\left[\boldsymbol{x}_{k} \mid \mathcal{I}_{k-1}\right],
\end{aligned}
$$

where $(*)$ is due to translation invariance property of entropy [31]. Applying Lemma 1 (see Appendix B) to $\mathrm{h}\left[\boldsymbol{x}_{k} \mid \mathcal{I}_{k-1}\right]$, we have

$$
\begin{aligned}
& \mathrm{h}\left[\boldsymbol{x}_{k} \mid \mathcal{I}_{k-1}\right]=\mathrm{h}\left[\boldsymbol{x}_{0} \mid \mathcal{I}_{k-1}\right]+ \\
& \sum_{j=1}^{M} \sum_{n=0}^{k-1} \mathrm{E}\left[\log \left(1+\mu_{n} \frac{\partial^{2}}{\partial x^{j^{2}}} U_{j}\left(x_{n}^{j}, \mathcal{D}_{n}\left(\boldsymbol{x}_{n}^{-j}\right)\right)\right) \mid \mathcal{I}_{k-1}\right]
\end{aligned}
$$

Using (17), E $\left[\mathrm{h}\left[\boldsymbol{x}_{k} \mid \mathcal{I}_{k-1}\right]\right]$ can be lower bounded as $\mathrm{E}\left[\mathrm{h}\left[\boldsymbol{x}_{k} \mid \mathcal{I}_{k-1}\right]\right]$

$$
\begin{aligned}
& =\sum_{j=1}^{M} \sum_{n=0}^{k-1} \mathrm{E}\left[\log \left(1+\mu_{n} \frac{\partial^{2}}{\partial x^{j}} U_{j}\left(x_{n}^{j}, \mathcal{D}_{n}\left(\boldsymbol{x}_{n}^{-j}\right)\right)\right)\right] \\
& \quad+\mathrm{E}\left[\mathrm{h}\left[\boldsymbol{x}_{0} \mid \mathcal{I}_{k-1}\right]\right] \\
& \stackrel{(*)}{\geq} \sum_{j=1}^{M} \sum_{n=0}^{k-1} \mathrm{E}\left[\log \left(1+\mu_{n} \frac{\partial^{2}}{\partial x^{j^{2}}} U_{j}\left(x_{n}^{j}, \mathcal{D}_{n}\left(\boldsymbol{x}_{n}^{-j}\right)\right)\right)\right] \\
& \quad+\mathrm{h}\left[\boldsymbol{x}_{0}\right]-\sum_{i=0}^{k-1} \log \left|\mathcal{A}_{i}\right|
\end{aligned}
$$

where $(*)$ follows from the following inequality established in [13]

$$
\mathrm{E}\left[\mathrm{h}\left[\boldsymbol{x}_{0} \mid \mathcal{I}_{k-1}\right]\right] \geq \mathrm{h}\left[\boldsymbol{x}_{0}\right]-\sum_{i=0}^{k-1} \log \left|\mathcal{A}_{i}\right| .
$$

Using (18), (16), and the fact that $\boldsymbol{x}_{0}$ has a finite entropy, the error exponent can be lower bounded as

$$
\begin{aligned}
& \liminf _{k \longrightarrow \infty} \frac{1}{k} \log \mathrm{E}\left[\left\|\boldsymbol{\epsilon}_{k}\right\|_{2}^{2}\right] \geq \frac{2}{M}\left(\liminf _{k \longrightarrow \infty} \sum_{j=1}^{M} \frac{1}{k} \sum_{n=0}^{k-1}\right. \\
& \left.\mathrm{E}\left[\log \left(1+\mu_{n} \frac{\partial^{2}}{\partial x^{j^{2}}} U_{j}\left(x_{n}^{j}, \mathcal{D}_{n}\left(\boldsymbol{x}_{n}^{-j}\right)\right)\right)\right]-R_{\mathcal{D}}\right) .
\end{aligned}
$$

Using Lemma 2 (see Appendix B), (20) can be written as

$$
\begin{aligned}
\liminf _{k \rightarrow \infty} \frac{1}{k} & \log \mathrm{E}\left[\left\|\boldsymbol{\epsilon}_{k}\right\|_{2}^{2}\right] \\
& \geq \frac{2}{M}\left(\sum_{i=1}^{M} \log \left(1+\left.\mu^{\star} \frac{\partial^{2} U_{i}}{\partial x^{i^{2}}}\right|_{\boldsymbol{x}_{\mathrm{NE}}}\right)-R_{\mathcal{D}}\right) .
\end{aligned}
$$

which completes the proof.

\section{APPENDIX B \\ TWO LEMMAS}

Lemma 1: Let $\mathcal{I}_{k-1}=\left\{\mathcal{D}_{i}\left(\boldsymbol{x}_{i}\right)=\boldsymbol{a}_{i}\right\}_{i=0}^{k-1}, \boldsymbol{a}_{i} \in \mathcal{A}_{i}$, be a possible output of the quantization scheme $\mathcal{D}$ at time $i$. Then

$$
\begin{aligned}
& \mathrm{h}\left[\boldsymbol{x}_{n} \mid \mathcal{I}_{k-1}\right]=\mathrm{h}\left[\boldsymbol{x}_{n-1} \mid \mathcal{I}_{k-1}\right]+ \\
& \mathrm{E}\left[\sum_{j=1}^{M} \log \left(1+\mu_{n-1} \frac{\partial^{2}}{\partial x^{j^{2}}} U_{j}\left(x_{n-1}^{j}, \mathcal{D}_{n-1}\left(\boldsymbol{x}_{n-1}^{-j}\right)\right)\right) \mid \mathcal{I}_{k-1}\right]
\end{aligned}
$$

Proof: Let $p_{\boldsymbol{x}_{n}}\left(\boldsymbol{x} \mid \mathcal{I}_{k-1}\right)$ and $p_{\boldsymbol{x}_{n-1}}\left(\boldsymbol{x} \mid \mathcal{I}_{k-1}\right)$ represent the probability density functions (PDFs) of $\boldsymbol{x}_{n}$ and $\boldsymbol{x}_{n-1}$, respectively, conditioned on $\mathcal{I}_{k-1}$. To prove this lemma, first, we obtain an expression for $p_{\boldsymbol{x}_{n}}\left(\boldsymbol{x} \mid \mathcal{I}_{k-1}\right)$ in terms of $p_{\boldsymbol{x}_{n-1}}\left(\boldsymbol{x} \mid \mathcal{I}_{k-1}\right)$. Then, this expression will be used to prove the result of this lemma. Given $\mathcal{I}_{k-1}$, update equation (1) at time $n$ under the quantization scheme $\mathcal{D}$ can be written as

$$
x_{n}^{i}=x_{n-1}^{i}+\mu_{n-1} \frac{\partial}{\partial x^{i}} U_{i}\left(x_{n-1}^{i}, \boldsymbol{a}_{n-1}^{-i}\right), \forall i .
$$

Let $F_{i}\left(x^{i}\right)=x^{i}+\mu_{n-1} \frac{\partial}{\partial x^{i}} U_{i}\left(x^{i}, \boldsymbol{a}_{n-1}^{-i}\right)$ and $F(\boldsymbol{x})=$ $\left[F_{1}\left(x^{1}\right), \cdots, F_{M}\left(x^{M}\right)\right]^{\top}$. Since $0<\mu_{n}<\frac{1}{\max _{i}\left|c_{i}\right|}$ for all $n$, we have $0<1+\mu_{n-1} \frac{\partial^{2}}{\partial x^{i 2}} U_{i}\left(x_{i}, \boldsymbol{a}_{n-1}^{-i}\right)<1$ which implies that $F_{i}\left(x^{i}\right)$ is invertible for all $i$. Thus, the PDF of $\boldsymbol{x}_{n}$ given $\mathcal{I}_{k-1}$ can be written as

$p_{\boldsymbol{x}_{n}}\left(\boldsymbol{x} \mid \mathcal{I}_{k-1}\right)=\frac{1}{\operatorname{det} J_{F}\left[F^{-1}(\boldsymbol{x})\right]} p_{\boldsymbol{x}_{n-1}}\left(F^{-1}(\boldsymbol{x}) \mid \mathcal{I}_{k-1}\right)$,

where $J_{F}[\boldsymbol{x}]$ is Jacobian of $F(\boldsymbol{x})$ evaluated at $\boldsymbol{x}$. Using (23), the conditional entropy of $\boldsymbol{x}_{n}$ given $\mathcal{I}_{k-1}$ can be written as

$$
\begin{aligned}
& \text { h }\left[\boldsymbol{x}_{n} \mid \mathcal{I}_{k-1}\right] \\
& =\int \log \left(\operatorname{det} J_{F}\left[F^{-1}(\boldsymbol{x})\right]\right) \frac{1}{\operatorname{det} J_{F}\left[F^{-1}(\boldsymbol{x})\right]} \\
& p_{\boldsymbol{x}_{n-1}}\left(F^{-1}(\boldsymbol{x}) \mid \mathcal{I}_{k-1}\right) d \boldsymbol{x} \\
& -\int \log \left(p_{\boldsymbol{x}_{n-1}}\left(F^{-1}(\boldsymbol{x}) \mid \mathcal{I}_{k-1}\right)\right) \frac{1}{\operatorname{det} J_{F}\left[F^{-1}(\boldsymbol{x})\right]} \\
& p_{\boldsymbol{x}_{n-1}}\left(F^{-1}(\boldsymbol{x}) \mid \mathcal{I}_{k-1}\right) d \boldsymbol{x}, \\
& \stackrel{(*)}{=} \int \log \left(\operatorname{det} J_{F}[\boldsymbol{y}]\right) p_{\boldsymbol{x}_{n-1}}\left(\boldsymbol{y} \mid \mathcal{I}_{k-1}\right) d \boldsymbol{x} \\
& -\int \log \left(p_{\boldsymbol{x}_{n-1}}\left(\boldsymbol{y} \mid \mathcal{I}_{k-1}\right)\right) p_{\boldsymbol{x}_{n-1}}\left(\boldsymbol{y} \mid \mathcal{I}_{k-1}\right) d \boldsymbol{x}, \\
& =\sum_{j=1}^{M} \mathrm{E}\left[\log \left(1+\mu_{n-1} \frac{\partial^{2}}{\partial x^{j^{2}}} U_{j}\left(x_{n-1}^{j}, \mathcal{D}_{n-1}\left(\boldsymbol{x}_{n-1}^{-j}\right)\right)\right) \mid \mathcal{I}_{k-1}\right] \\
& +\mathrm{h}\left[\boldsymbol{x}_{n-1} \mid \mathcal{I}_{k-1}\right] \text {, }
\end{aligned}
$$


where $(*)$ follows from change of variable $\boldsymbol{y}=F^{-1}(\boldsymbol{x})$.

\section{Lemma 2:}

$$
\begin{gathered}
\lim _{k \longrightarrow \infty} \sum_{j=1}^{M} \frac{1}{k} \sum_{n=0}^{k-1} \mathrm{E}\left[\log \left(1+\mu_{n} \frac{\partial^{2}}{\partial x^{j^{2}}} U_{j}\left(x_{n}^{j}, \boldsymbol{x}_{n}^{-j}\right)\right)\right]= \\
\sum_{j=1}^{M} \log \left(1+\mu^{\star} \frac{\partial^{2}}{\partial x^{j^{2}}} U_{j}\left(x_{\mathrm{NE}}^{j}, \boldsymbol{x}_{\mathrm{NE}}^{-j}\right)\right) .
\end{gathered}
$$

Proof: For $\beta>0$, define the event $B_{n}$ as

$$
\begin{aligned}
B_{n}=\left\{\left\|\boldsymbol{x}_{n}-\boldsymbol{x}_{\mathrm{NE}}\right\|_{2} \leq \beta,\right. \\
\left.\left\|\mathcal{D}_{n}\left(\boldsymbol{x}_{n}\right)-\boldsymbol{x}_{\mathrm{NE}}\right\|_{2} \leq \beta,\left|\mu_{n}-\mu^{\star}\right| \leq \beta\right\} .
\end{aligned}
$$

Also, let's define the set $B$ as

$B=\left\{\left\|\boldsymbol{x}-\boldsymbol{x}_{\mathrm{NE}}\right\|_{2} \leq \beta,\left\|\hat{\boldsymbol{x}}-\boldsymbol{x}_{\mathrm{NE}}\right\|_{2} \leq \beta,\left|\mu-\mu^{\star}\right| \leq \beta\right\}$.

Then, we have

$\frac{1}{k} \sum_{n=0}^{k-1} \sum_{j=1}^{M} \mathrm{E}\left[\log \left(1+\mu_{n} \frac{\partial^{2}}{\partial x^{j^{2}}} U_{j}\left(x_{n}^{j}, \boldsymbol{x}_{n}^{-j}\right)\right)\right]$

$=\frac{1}{k} \sum_{n=0}^{k-1} \sum_{j=1}^{M} \mathrm{E}\left[\log \left(1+\mu_{n} \frac{\partial^{2}}{\partial x^{j^{2}}} U_{j}\left(x_{n}^{j}, \boldsymbol{x}_{n}^{-j}\right)\right)\left(1_{\left\{B_{n}\right\}}+1_{\left\{B_{n}^{c}\right\}}\right)\right]$,

$\inf _{\{\boldsymbol{x}, \hat{\boldsymbol{x}}, \mu\} \in B} \sum_{j=1}^{M} \log \left(1+\mu \frac{\partial^{2}}{\partial x^{j^{2}}} U_{j}\left(x^{j}, \hat{\boldsymbol{x}}^{-j}\right)\right) \mathrm{E}\left[\frac{1}{k} \sum_{n=0}^{k-1} 1_{\left\{B_{n}\right\}}\right]$

$$
+\sum_{j=1}^{M} \log \left(1+\inf _{n} \mu_{n} c_{j}\right) \mathrm{E}\left[\frac{1}{k} \sum_{n=0}^{k-1} 1_{\left\{B_{n}^{c}\right\}}\right],
$$

where $B_{n}^{c}$ is the complement of $B_{n}$. Since, $\lim _{k \rightarrow \infty} \boldsymbol{x}_{k}=$ $\boldsymbol{x}_{\mathrm{NE}}$ and $\lim _{k \longrightarrow \infty} \mathcal{D}_{k}\left(\boldsymbol{x}_{k}\right)=\boldsymbol{x}_{\mathrm{NE}}$ for any initial vector of agents' actions, i.e., $\boldsymbol{x}_{0}$, we have $1_{\left\{B_{n}\right\}} \longrightarrow 1$ almost surely and $\frac{1}{k} \sum_{n=0}^{k-1} 1_{\left\{B_{n}\right\}} \longrightarrow 1$ almost surely. Thus, using Fatou's Lemma, we have $\liminf _{k \rightarrow \infty} \mathrm{E}\left[\frac{1}{k} \sum_{n=0}^{k-1} 1_{\left\{B_{n}\right\}}\right] \geq$ 1. Similarly, we have $\frac{1}{k} \sum_{n=0}^{k-1} 1_{\left\{B_{n}^{c}\right\}} \longrightarrow 0$ almost surely. Using, Lebesgue dominated convergence Theorem, we have $\lim _{k \rightarrow \infty} \mathrm{E}\left[\frac{1}{k} \sum_{n=0}^{k-1} 1_{\left\{B_{n}^{c}\right\}}\right]=0$. Thus, we have

$$
\begin{gathered}
\liminf _{k \longrightarrow \infty} \frac{1}{k} \sum_{n=0}^{k-1} \sum_{j=1}^{M} \mathrm{E}\left[\log \left(1+\mu_{n} \frac{\partial^{2}}{\partial x^{j^{2}}} U_{j}\left(x_{n}^{j}, \boldsymbol{x}_{n}^{-j}\right)\right)\right] \geq \\
\inf _{\{\boldsymbol{x}, \hat{\boldsymbol{x}}, \mu\} \in B} \sum_{j=1}^{M} \log \left(1+\mu \frac{\partial^{2}}{\partial x^{j^{2}}} U_{j}\left(\boldsymbol{x}^{j}, \hat{\boldsymbol{x}}^{-j}\right)\right) .
\end{gathered}
$$

Since, $\beta$ is an arbitrary positive real number, we have

$$
\begin{gathered}
\liminf _{k \longrightarrow \infty} \frac{1}{k} \sum_{n=0}^{k-1} \sum_{j=1}^{M} \mathrm{E}\left[\log \left(1+\mu_{n} \frac{\partial^{2}}{\partial x^{j^{2}}} U_{j}\left(x_{n}^{j}, \boldsymbol{x}_{n}^{-j}\right)\right)\right] \geq \\
\left.\sum_{j=1}^{M} \log \left(1+\mu^{\star} \frac{\partial^{2}}{\partial x^{j^{2}}} U_{j}\left(x^{j}, \boldsymbol{x}^{-j}\right)\right)\right|_{\boldsymbol{x}_{\mathrm{NE}}} .
\end{gathered}
$$

To show the other side, note that

$$
\begin{aligned}
& \frac{1}{k} \sum_{n=0}^{k-1} \sum_{j=1}^{M} \mathrm{E}\left[\log \left(1+\mu_{n} \frac{\partial^{2}}{\partial x^{j^{2}}} U_{j}\left(x_{n}^{j}, \boldsymbol{x}_{n}^{-j}\right)\right)\right] \leq \\
& \sup _{\{\boldsymbol{x}, \hat{\boldsymbol{x}}, \mu\} \in B} \sum_{j=1}^{M} \log \left(1+\mu \frac{\partial^{2}}{\partial x^{j^{2}}} U_{j}\left(x^{j}, \hat{\boldsymbol{x}}^{-j}\right)\right) \mathrm{E}\left[\frac{1}{k} \sum_{n=0}^{k-1} 1_{\left\{B_{n}\right\}}\right] \\
& +\sum_{j=1}^{M} \log \left(1+\sup _{n} \mu_{n} b_{j}\right) \mathrm{E}\left[\frac{1}{k} \sum_{n=0}^{k-1} 1_{\left\{B_{n}^{c}\right\}}\right] .
\end{aligned}
$$

Using similar argument as above, we can show that

$$
\begin{aligned}
\limsup _{k \rightarrow \infty} & \frac{1}{k} \sum_{n=0}^{k-1} \sum_{j=1}^{M} \mathrm{E}\left[\log \left(1+\mu_{n} \frac{\partial^{2}}{\partial x^{j^{2}}} U_{j}\left(x_{n}^{j}, \boldsymbol{x}_{n}^{-j}\right)\right)\right] \leq \\
& \sum_{j=1}^{M} \log \left(1+\mu^{\star} \frac{\partial^{2}}{\partial x^{j^{2}}} U_{j}\left(x_{\mathrm{NE}}^{j}, \boldsymbol{x}_{\mathrm{NE}}^{-i}\right)\right),
\end{aligned}
$$

which completes the proof.

\section{APPENDIX C}

PROOF OF THEOREM 2

$\frac{\partial}{\partial x^{i}} U_{i}\left(x^{i}, \mathcal{D}_{u}\left(\boldsymbol{x}_{k}^{-i}\right)\right) \frac{\partial}{\partial x^{i}} U_{i}\left(x_{k}^{i}, \mathcal{D}_{u}\left(\boldsymbol{x}_{k}^{-i}\right)\right)$ In this appendix, first, it is shown that the sequence of updates $\left\{\boldsymbol{x}_{k}\right\}_{k=0}^{\infty}$, generated by (7), will always stay in $\mathrm{Q}\left(\boldsymbol{x}_{c}, 3 d / 2\right)$, hence, the quantization scheme $\mathcal{D}_{\mathrm{u}}$ is always well defined. Later, the upper bound on the expected time required to settle inside $\mathrm{B}\left(\boldsymbol{x}_{\mathrm{NE}}, r\right)$ is derived. Next lemma shows that the quantization scheme $\mathcal{D}_{\mathrm{u}}$ is well defined.

Lemma 3: Suppose that the quantization step, $\delta$, satisfies (8), and $\boldsymbol{x}_{0}$ belongs to $\mathcal{R}$, then, sequence of updates $\left\{\boldsymbol{x}_{k}\right\}_{k=0}^{\infty}$ generated by (7) will always stay in $\mathrm{Q}\left(\boldsymbol{x}_{c}, 3 d / 2\right)$.

Proof: Since $\boldsymbol{x}_{0}$ belongs to $\mathcal{R}$ and $\mathcal{R} \subset \mathrm{B}\left(\boldsymbol{x}_{\mathrm{NE}}, d\right) \subset$ $\mathrm{Q}\left(\boldsymbol{x}_{c}, 3 d / 2\right)$, it suffices to show that if $\boldsymbol{x}_{k}$ belongs to $\mathrm{B}\left(\boldsymbol{x}_{\mathrm{NE}}, d\right)$, then $\boldsymbol{x}_{k+1}$ belongs to $\mathrm{B}\left(\boldsymbol{x}_{\mathrm{NE}}, d\right)$. To prove this result, we derive an upper bound on the distance between actions of agents at time $(k+1)$ and the NE as follows.

Let $\mathcal{D}_{\mathrm{u}}\left(\boldsymbol{x}_{k}\right)$ and $\mathcal{D}_{\mathrm{u}}\left(\boldsymbol{x}_{k}^{-i}\right)$ represent the quantized versions of $\boldsymbol{x}_{k}$ and $\boldsymbol{x}_{k}^{-i}$, respectively. Under the quantization scheme $\mathcal{D}_{\mathrm{u}}$, at each time, each agent will update its action based on the quantized version of other agents' actions. Therefore, the action of the $i$-th agent in time $k+1$, under the quantization scheme $\mathcal{D}_{\mathrm{u}}$, can be written as

$$
\begin{aligned}
x_{k+1}^{i} & =x_{k}^{i}+\mu_{k} \frac{\partial}{\partial x^{i}} U_{i}\left(x_{k}^{i}, \mathcal{D}_{\mathrm{u}}\left(\boldsymbol{x}_{k}^{-i}\right)\right) \\
& =x_{k}^{i}+\mu_{k} \frac{\partial}{\partial x^{i}} U_{i}\left(x_{k}^{i}, \boldsymbol{x}_{k}^{-i}\right) \\
& +\mu_{k}\left[\frac{\partial}{\partial x^{i}} U_{i}\left(x_{k}^{i}, \mathcal{D}_{\mathrm{u}}\left(\boldsymbol{x}_{k}^{-i}\right)\right)-\frac{\partial}{\partial x^{i}} U_{i}\left(x_{k}^{i}, \boldsymbol{x}_{k}^{-i}\right)\right] .
\end{aligned}
$$

Let $\boldsymbol{\delta}_{k}=\boldsymbol{x}_{k}-\mathcal{D}_{\mathrm{u}}\left(\boldsymbol{x}_{k}\right)$ be the vector of quantization error at time $k$. Then, using the Taylor expansion [32] of 
$\frac{\partial}{\partial x^{i}} U_{i}\left(x^{i}, \boldsymbol{x}^{-i}\right)$ at $\mathcal{D}_{\mathrm{u}}\left(\boldsymbol{x}_{k}\right)$, we can write $\frac{\partial}{\partial x^{i}} U_{i}\left(x_{k}^{i}, \boldsymbol{x}_{k}^{-i}\right)$ as

$$
\begin{aligned}
\frac{\partial}{\partial x^{i}} U_{i} & \left(x_{k}^{i}, \boldsymbol{x}_{k}^{-i}\right)=\frac{\partial}{\partial x^{i}} U_{i}\left(\mathcal{D}_{\mathrm{u}}\left(x_{k}^{i}\right), \mathcal{D}_{\mathrm{u}}\left(\boldsymbol{x}_{k}^{-i}\right)\right) \\
& +\frac{\partial^{2}}{\partial x^{i^{2}}} U_{i}\left(\mathcal{D}_{\mathrm{u}}\left(x_{k}^{i}\right), \mathcal{D}_{\mathrm{u}}\left(\boldsymbol{x}_{k}^{-i}\right)\right) \delta_{k}^{i} \\
& +\sum_{j \neq i} \frac{\partial^{2}}{\partial^{2} x^{j} x^{i}} U_{i}\left(\mathcal{D}_{\mathrm{u}}\left(x_{k}^{i}\right), \mathcal{D}_{\mathrm{u}}\left(\boldsymbol{x}_{k}^{-i}\right)\right) \delta_{k}^{j} \\
& +\left.\frac{1}{2} \boldsymbol{\delta}_{k}^{\top} \nabla^{2} \frac{\partial}{\partial x^{i}} U_{i}\left(x^{i}, \boldsymbol{x}^{-i}\right)\right|_{\beta \boldsymbol{x}_{k}+(1-\beta) \mathcal{D}_{\mathrm{u}}\left(\boldsymbol{x}_{k}\right)} \boldsymbol{\delta}_{k},
\end{aligned}
$$

where $\beta \in[0,1], \delta_{k}^{j}$ is th $j$-th element of $\boldsymbol{\delta}_{k}$ and $\nabla^{2}(\cdot)$ represents the Hessian operator. Similarly, using the Taylor expansion of $\frac{\partial}{\partial x^{i}} U_{i}\left(x^{i}, \mathcal{D}_{\mathrm{u}}\left(\boldsymbol{x}_{k}^{-i}\right)\right)$ at $\mathcal{D}_{\mathrm{u}}\left(\boldsymbol{x}_{k}^{i}\right)$, we can express $\frac{\partial}{\partial x^{i}} U_{i}\left(x_{k}^{i}, \mathcal{D}_{\mathrm{u}}\left(\boldsymbol{x}_{k}^{-i}\right)\right)$ as

$$
\begin{aligned}
\frac{\partial}{\partial x^{i}} U_{i} & \left(x_{k}^{i}, \mathcal{D}_{\mathrm{u}}\left(\boldsymbol{x}_{k}^{-i}\right)\right)=\frac{\partial}{\partial x^{i}} U_{i}\left(\mathcal{D}_{\mathrm{u}}\left(x_{k}^{i}\right), \mathcal{D}_{\mathrm{u}}\left(\boldsymbol{x}_{k}^{-i}\right)\right) \\
& +\frac{\partial^{2}}{\partial x^{i^{2}}} U_{i}\left(\mathcal{D}_{\mathrm{u}}\left(x_{k}^{i}\right), \mathcal{D}_{\mathrm{u}}\left(\boldsymbol{x}_{k}^{-i}\right)\right) \delta_{k}^{i} \\
& +\frac{1}{2} \frac{\partial^{3}}{\partial x^{i^{3}}} U_{i}\left(\beta^{\prime} x_{k}^{i}+\left(1-\beta^{\prime}\right) \mathcal{D}_{\mathrm{u}}\left(x_{k}^{i}\right), \mathcal{D}_{\mathrm{u}}\left(\boldsymbol{x}_{k}^{-i}\right)\right)\left(\delta_{k}^{i}\right)^{2},
\end{aligned}
$$

where $\beta^{\prime} \in[0,1]$. Using (25) and (26), the update rule under quantization scheme $\mathcal{D}_{\mathrm{u}}$ can be written as

$$
\begin{aligned}
& x_{k+1}^{i}=x_{k}^{i}+\mu_{k} \frac{\partial}{\partial x^{i}} U_{i}\left(x_{k}^{i}, \boldsymbol{x}_{k}^{-i}\right) \\
& -\mu_{k} \sum_{j \neq i} \frac{\partial^{2}}{\partial^{2} x^{j} x^{i}} U_{i}\left(\mathcal{D}_{\mathrm{u}}\left(x_{k}^{i}\right), \mathcal{D}_{\mathrm{u}}\left(\boldsymbol{x}_{k}^{-i}\right)\right) \delta_{k}^{j} \\
& -\left.\frac{\mu_{k}}{2} \boldsymbol{\delta}_{k}^{\top} \nabla^{2} \frac{\partial}{\partial x^{i}} U_{i}\left(x^{i}, \boldsymbol{x}^{-i}\right)\right|_{\beta \boldsymbol{x}_{k}+(1-\beta) \mathcal{D}_{\mathrm{u}}\left(\boldsymbol{x}_{k}\right)} \boldsymbol{\delta}_{k} \\
& +\frac{\mu_{k}}{2} \frac{\partial^{3}}{\partial x^{i^{3}}} U_{i}\left(\beta^{\prime} x_{k}^{i}+\left(1-\beta^{\prime}\right) \mathcal{D}_{\mathrm{u}}\left(x_{k}^{i}\right), \mathcal{D}_{\mathrm{u}}\left(\boldsymbol{x}_{k}^{-i}\right)\right)\left(\delta_{k}^{i}\right)^{2},
\end{aligned}
$$

Applying the triangle inequality to (27) and using the fact that the unquantized update rule is a pseudo-contraction, the distance between the action of agents and $\mathrm{NE}$ at time $k+1$ can be upper bounded as

$$
\begin{gathered}
\left\|\boldsymbol{x}_{k+1}-\boldsymbol{x}_{\mathrm{NE}}\right\|_{2} \leq \alpha\left\|\boldsymbol{x}_{k}-\boldsymbol{x}_{\mathrm{NE}}\right\|_{2}+ \\
\mu_{k}\left(\delta \sqrt{\sum_{i} \Phi_{i}^{2}}+\frac{1}{2} \delta^{2}\left(M \sqrt{\sum_{i} \Psi_{i}^{2}}+\sqrt{\sum_{i} \eta_{i}^{2}}\right)\right), \\
\leq \alpha\left\|\boldsymbol{x}_{k}-\boldsymbol{x}_{\mathrm{NE}}\right\|_{2}+ \\
\sup _{k} \mu_{k}\left(\delta \sqrt{\sum_{i} \Phi_{i}^{2}}+\frac{1}{2} \delta^{2}\left(M \sqrt{\sum_{i} \Psi_{i}^{2}}+\sqrt{\sum_{i} \eta_{i}^{2}}\right)\right), \\
\leq \alpha d+(1-\alpha) d=d .
\end{gathered}
$$

where $\Phi_{i}, \Psi_{i}$ and $\eta_{i}$ are given by

$$
\begin{aligned}
\Phi_{i} & =\sup _{\boldsymbol{x} \in \mathrm{Q}\left(\boldsymbol{x}_{c}, 3 d / 2\right)} \sum_{j \neq i}\left|\frac{\partial^{2}}{\partial^{2} x^{j} x^{i}} U_{i}\left(x^{i}, \boldsymbol{x}^{-i}\right)\right|, \\
\Psi_{i} & =\sup _{\boldsymbol{x} \in \mathrm{Q}\left(\boldsymbol{x}_{c}, 3 d / 2\right)}\left\|\nabla^{2} \frac{\partial}{\partial x^{i}} U_{i}\left(x^{i}, \boldsymbol{x}^{-i}\right)\right\|_{2}, \\
\eta_{i} & =\sup _{\boldsymbol{x} \in \mathrm{Q}\left(\boldsymbol{x}_{c}, 3 d / 2\right)}\left|\frac{\partial^{3}}{\partial x^{i^{3}}} U_{i}\left(x^{i}, \boldsymbol{x}^{-i}\right)\right|,
\end{aligned}
$$

respectively. Inequality (29) implies that $\boldsymbol{x}_{k+1}$ belongs to $\mathrm{B}\left(\boldsymbol{x}_{\mathrm{NE}}, d\right) \subset \mathrm{Q}\left(\boldsymbol{x}_{c}, 3 d / 2\right)$ which completes the proof.

Now, we are ready to prove Theorem 2 . To this end, we use (28) to upper bound the distance between the NE and the actions of agents at time $(k+1)$ as follows:

$$
\begin{aligned}
& \left\|\boldsymbol{x}_{k+1}-\boldsymbol{x}_{\mathrm{NE}}\right\|_{2} \leq \alpha^{k+1}\left\|\boldsymbol{x}_{0}-\boldsymbol{x}_{\mathrm{NE}}\right\|_{2}+ \\
& \frac{\sup _{k} \mu_{k}}{1-\alpha}\left(\delta \sqrt{\sum_{i} \Phi_{i}^{2}}+\frac{1}{2} \delta^{2}\left(M \sqrt{\sum_{i} \Psi_{i}^{2}}+\sqrt{\sum_{i} \eta_{i}^{2}}\right)\right),
\end{aligned}
$$

For a given initial value of actions of agents, let $\mathcal{N}^{u}$ be the number of time-steps for the upper bound in (30) to enter $\mathrm{B}\left(\boldsymbol{x}_{\mathrm{NE}}, r\right)$. Clearly, we have $\mathcal{N} \leq \mathcal{N}^{\mathrm{u}}$. For a given $\boldsymbol{x}_{0}, \mathcal{N}^{\mathrm{u}}$ can be upper bounded, using (28), as

$$
\begin{aligned}
\mathcal{N}^{\mathrm{u}} & \leq\left\lceil\frac{\log \left\|\boldsymbol{x}_{0}-\boldsymbol{x}_{\mathrm{NE}}\right\|_{2}-\log (r-\theta)}{\log \frac{1}{\alpha}}-1\right]^{+}, \\
& \leq \frac{1}{\log \frac{1}{\alpha}}\left(\log \left(\frac{\left\|\boldsymbol{x}_{0}-\boldsymbol{x}_{\mathrm{NE}}\right\|_{2}}{r-\theta}\right) 1_{\left\{\frac{\| \boldsymbol{x}_{0}-\boldsymbol{x}_{\mathrm{NE}}}{r-\theta} \|_{2} \geq 1\right\}}\right),
\end{aligned}
$$

where $\lceil x\rceil=\max (0, x)$. Thus, we have

$\mathrm{E}[\mathcal{N}] \leq$

$$
\left.\frac{1}{\log \left(\frac{1}{\alpha}\right)}\left(\mathrm{E}\left[\log \left(\frac{\left\|\boldsymbol{x}_{0}-\boldsymbol{x}_{\mathrm{NE}}\right\|_{2}}{r-\theta}\right) 1_{\left\{\frac{\left\|\boldsymbol{x}_{0}-\boldsymbol{x}_{\mathrm{NE}}\right\|_{2}}{r-\theta}>1\right.}\right\}\right]\right) .
$$

\section{APPENDIX D}

\section{Proof of THEOREM 3}

Using (30), the probability that $\boldsymbol{x}_{k}$ lies outside B $\left(\boldsymbol{x}_{\mathrm{NE}}, r\right)$ can be upper bounded as

$\operatorname{Pr}\left\{\left\|\boldsymbol{x}_{k}-\boldsymbol{x}_{\mathrm{NE}}\right\|_{2}>r\right\} \leq \operatorname{Pr}\left\{\left\|\boldsymbol{x}_{0}-\boldsymbol{x}_{\mathrm{NE}}\right\|_{2}>\frac{r-\theta}{\alpha^{k}}\right\}$.

Also, using the Chernoff inequality, $\operatorname{Pr}\left\{\left\|\boldsymbol{x}_{0}-\boldsymbol{x}_{\mathrm{NE}}\right\|_{2}>\frac{r-\theta}{\alpha^{k}}\right\}$ can be upper bounded as

$\operatorname{Pr}\left\{\left\|\boldsymbol{x}_{0}-\boldsymbol{x}_{\mathrm{NE}}\right\|_{2}>\frac{r-\theta}{\alpha^{k}}\right\} \leq \mathrm{e}^{-\frac{r-\theta}{\alpha^{k}}} \mathrm{E}\left[\mathrm{e}^{\left\|\boldsymbol{x}_{0}-\boldsymbol{x}_{\mathrm{NE}}\right\|_{2}}\right]$.

Since probability is less than one, we have

$$
\begin{aligned}
& \operatorname{Pr}\left\{\left\|\boldsymbol{x}_{k}-\boldsymbol{x}_{\mathrm{NE}}\right\|_{2}>r\right\} \leq \\
& \min \left(1, \frac{r-\theta}{\alpha^{k}} \leq \mathrm{e}^{-\frac{r-\theta}{\alpha^{k}}} \mathrm{E}\left[\mathrm{e}^{\left\|\boldsymbol{x}_{0}-\boldsymbol{x}_{\mathrm{NE}}\right\|_{2}}\right]\right)
\end{aligned}
$$




\section{REFERENCES}

[1] C. U. Saraydar, N. B. Mandayam, D. Goodman, "Efficient power control via pricing in wireless data networks," IEEE Trans. Commun., vol. 50, no. 2, pp. 291-303, Feb 2002.

[2] R. J. Marden, J. S. Shamma, Game theory and distributed control, Handbook of game theory, Edited by P. Young, S. Zamir, Burlington: Elsevier Science, 2014.

[3] N. D. Stein, Characterization and Computation of Equilibria in Infinite Games, Master Thesis, M.I.T., Jun., 2007.

[4] $\mathrm{S}$. Li and $\mathrm{T}$. Basar, "Distributed algorithms for the computation of noncooperative equilibria", Automatica, vol. 23, pp. 523533, 1987.

[5] A. Nedić and D.P. Bertsekas, "Incremental Subgradient Methods for Nondifferentiable Optimization", SIAM J. Control Optim., vol. 12, no. 1, pp. 1091382001.

[6] A. Nedić and A. Ozdaglar, "Distributed Subgradient Methods for MultiAgent Optimization," IEEE Trans. Autom. Control, vol. 54, no. 1, pp. 4861, Jan. 2009.

[7] A. Nedić, A. Ozdaglar and P.A. Parrilo, "Constrained Consensus and Optimization in Multi-Agent Networks," IEEE Trans. Autom. Control, vol. 55, no. 4, pp. 922-938, April 2010.

[8] A. Nedić, A. Olshevsky, A. Ozdaglar and J.N. Tsitsiklis, "Distributed subgradient methods and quantization effects," IEEE Conf. on Decision and Control Conference (CDC), pp.4177,4184, 9-11 Dec. 2008.

[9] M.G. Rabbat and R.D. Nowak, "Quantized incremental algorithms for distributed optimization," IEEE J. Sel. Areas. Comm., vol. 23, no. 4, pp. 798,808, April 2005.

[10] Y. Cui, and V.K.N. Lau, "Convergence-optimal quantizer design of distributed contraction-based iterative algorithms with quantized message passing.", IEEE Trans. Signal Process., vol. 58, no. 10, pp. 5196-5205, Oct. 2010.

[11] R. Dong, and Z. Geng, "Design and analysis of quantizer for multi-agent systems with a limited rate of communication data," Communications in Nonlinear Science and Numerical Simulation, pp. 282-290, vol. 18, no. 2, 2013.

[12] R. Carli, F. Fagnani, A. Speranzon and S. Zampieri, "Communication constraints in the average consensus problem," Automatica, vol. 44, no. 3 , pp. 671-684, 2008.

[13] G.N. Nair and R.J. Evans, "Stabilizability of stochastic linear systems with finite feedback data rates," SIAM J. Control Optim., vol. 43, no. 2, pp. 413-436, 2004.

[14] G.N. Nair, F. Fagnani, S. Zampieri, and R.J. Evans, "Feedback Control Under Data Rate Constraints: An Overview," Proc. IEEE, vol. 95, no. 1, pp. 108-137, Jan. 2007

[15] A. Kashyap, T. Baar and R. Srikant, "Quantized consensus," Automatica, vol. 43, no. 7 pp. 1192-1203, 2007

[16] A. Nedić, A. Olshevsky, A. Ozdaglar and J. N. Tsitsiklis, "On distributed averaging algorithms and quantization effects," IEEE Trans. Autom. Control, vol. 54, no. 11, pp. 2506-2517, Nov. 2009.

[17] K. You and L. Xie, "Network Topology and Communication Data Rate for Consensusability of Discrete-Time Multi-Agent Systems," IEEE Trans. Autom. Control, vol. 56, no. 10, pp. 2262-2275, Oct. 2011.

[18] J. B. Rosen, "Existence and uniqueness of equilibrium points for concave n-person games," Econometrica: Journal of the Econometric Society, pp. 520-534, 1965

[19] T. Alpcan, Noncooperative games for control of networked systems, PhD Dissertation, University of Illinois at Urbana-Champaign, Urbana, IL, February 2006.

[20] D. Chattopadhyay and T. Alpcan, "A Game-Theoretic Analysis of Wind Generation Variability on Electricity Markets," IEEE Trans. on Power Systems, vol. 29, no. 5, pp. 2069-2077, Sept. 2014.

[21] E. Nekouei, T. Alpcan and D. Chattopadhyay, "Game-Theoretic Frameworks for Demand Response in Electricity Markets," IEEE Trans. on Smart Grid, vol. 6, no. 2, pp. 748-758, March 2015.

[22] D. Chattopadhyay, "Multicommodity spatial Cournot model for generator bidding analysis," IEEE Trans. on Power Systems, vol. 19, no. 1 , pp. 267-275, 2004.

[23] P. Minero, M. Franceschetti, S. Dey and G.N. Nair, "Data Rate Theorem for Stabilization Over Time-Varying Feedback Channels," IEEE Trans. Autom. Control, vol. 54, no. 2, pp.243-255, Feb. 2009.

[24] J.S. Freudenberg, R.H. Middleton, V. Solo, "Stabilization and Disturbance Attenuation Over a Gaussian Communication Channel," IEEE Trans. Autom. Control, vol. 55, no. 3, pp.795-799, March 2010.

[25] F. D. Delchamps, "Stabilizing a linear system with quantized state feedback," IEEE Transactions on Automatic Control, vol. 35, no. 8, pp. 916-924, Aug. 1990
[26] M. S. Stankovic, K. H. Johansson, and D. M. Stipanovic, "Distributed seeking of Nash equilibria with applications to mobile sensor networks," IEEE Trans. Autom. Control, vol. 57, no. 4, pp. 904-919, 2012.

[27] S. Liu and M. Krstic. Stochastic, "Nash equilibrium seeking for games with general nonlinear payoffs," SIAM Journal on Control and Optimization, pp. 49, vol. 4, pp. 1659-1679, 2011.

[28] P. Frihauf, M. Krstic, and T. Basar, "Nash equilibrium seeking in non-cooperative games,", IEEE Trans. Autom. Control, vol. 57, no. 5, pp. 1192-1207, 2011.

[29] H.-B. Durr, M. S. Stankovic, and K. H. Johansson, "Distributed positioning of autonomous mobile sensors with application to coverage control,", Proc. Amer. Control Conf., 2011.

[30] S. Boyd and L. Vandenberghe, Convex Optimization, Cambridge University Press, New York, 2004.

[31] T. M. Cover and J. A. Thomas, "Elements of Information Theory", New York: Wiley, 2005

[32] D. P. Bertsekas and J. N. Tsitsiklis, Parallel and Distributed Computation: Numerical Methods, New jersey: Prentice-Hall, 1989.

Ehsan Nekouei (S11, M14 ) received B.Sc. from Shahid Bahonar University of Kerman, Kerman, Iran, in 2003 and M.Sc. form Tarbiat Modares University, Tehran, Iran, in 2006. He received Ph.D. degree in Electrical Engineering from The University of Melbourne in 2013. He is currently working as a research fellow in the Department of Electrical and Electronic Engineering, University of Melbourne. His research interests include game theory, information theory and dynamical systems.

Girish N. Nair was born in Malaysia and obtained a B.E. (Elec., 1st class hons.) in 1994, B.Sc. (math.) in 1995, and Ph.D. (elec. eng.) in 2000, on scholarships from the Australian government and Uni. Melbourne. He is currently a Professor in the Dept. Electrical and Electronic Engineering at Uni. Melbourne. He has received several prizes, including the IEEE CSS George S. Axelby Outstanding Paper Award in 2014, a SIAM Outstanding Paper Prize in 2006, and the Best Theory Paper Prize at the UKACC Int. Conf. Control, Cambridge Uni., 2000.

Tansu Alpcan Tansu Alpcan (SM13) received his B.S. degree in electrical engineering from Bogazici University, Istanbul, Turkey in 1998. He received his M.S. and Ph.D. degrees in Electrical and Computer Engineering from University of Illinois at Urbana- Champaign in 2001 and 2006, respectively. His research involves applications of distributed decision making, game theory, optimization, and control to various security and resource allocation problems in networked and energy systems. He is recipient of multiple research and best paper awards from UIUC and IEEE. He has played a role in organization of several workshops and conferences such as IEEE Infocom, ICC, GameComm, and GameSec as TPC member, associate editor, co-chair, chair, and steering board member. He is the (co-)author of more than 130 journal and conference articles, two edited volumes, as well as the book Network Security: A Decision and Game Theoretic Approach published by the Cambridge University Press in 2011. He has worked as a Senior Research Scientist in Deutsche Telekom Laboratories, Berlin, Germany, between 20062009, and as Assistant Professor in Technical University of Berlin from 2009 until 2011. He is currently with the Department of Electrical and Electronic Engineering at the University of Melbourne as Associate Professor and Reader. 


\section{University Library}

\section{- M M N E R VA A gateway to Melbourne's research publications}

Minerva Access is the Institutional Repository of The University of Melbourne

Author/s:

Nekouei, E;Nair, GN;Alpcan, T

Title:

Performance Analysis of Gradient-Based Nash Seeking Algorithms Under Quantization

Date:

2016-12-01

Citation:

Nekouei, E., Nair, G. N. \& Alpcan, T. (2016). Performance Analysis of Gradient-Based Nash Seeking Algorithms Under Quantization. IEEE TRANSACTIONS ON AUTOMATIC CONTROL, 61 (12), pp.3771-3783. https://doi.org/10.1109/TAC.2016.2526598.

Persistent Link:

http://hdl.handle.net/11343/241545 